\title{
Cooperation through social influence
}

\author{
Xavier Molinero $^{\mathrm{a}, 1}$, Fabián Riquelme ${ }^{\mathrm{b}, 2}$, Maria Serna ${ }^{\mathrm{b}, 3}$ \\ ${ }^{a}$ Department of Applied Mathematics III. Universitat Politècnica de Catalunya, Manresa, Spain \\ ${ }^{b}$ Department of Computer Science, Universitat Politècnica de Catalunya, Barcelona, Spain
}

\begin{abstract}
We consider a simple and altruistic multiagent system in which the agents are eager to perform a collective task but where their real engagement depends on the willingness to perform the task of other influential agents. We model this scenario by an influence game, a cooperative simple game in which a team (or coalition) of players succeeds if it is able to convince enough agents to participate in the task (to vote in favor of a decision). We take the linear threshold model as the influence model. We show first the expressiveness of influence games showing that they capture the class of simple games. Then we characterize the computational complexity of various problems on influence games, including measures (length and width), values (Shapley-Shubik and Banzhaf) and properties (of teams and players). Finally, we analyze those problems for some particular extremal cases, with respect to the propagation of influence, showing tighter complexity characterizations.
\end{abstract}

Keywords: Spread of influence, Simple games, Influence games, Computational complexity

\section{Introduction}

Cooperation towards task execution when tasks cannot be performed by a single agent is one of the fundamental problems in both social and multiagent systems. There has been a lot of research understanding collective tasks allocation under different models coming from cooperative game theory. Under such framework, in general, cooperation is achieved by splitting the agents into teams so that each team performs a particular task and the pay-off of the team is split among the team members. Thus, cooperative game theory provides the fundamental tools to analyze this context. Among the many references we point the reader to [55, 56, 13, 43, 6, 15].

The ways in which people influence each other through their interactions in a social network has received a lot of attention in the last decade. Social networks have become a huge interdisciplinary research area with important links to sociology, economics, epidemiology, computer science, and mathematics [37, 19, 2, 34] (players face the choice of adopting a specific product or not; users choose among competing programs from providers of mobile telephones, having the option to adopt more than one product at an extra cost; etc.). A social network can be represented by a graph where each node is an agent and each edge represents the degree of influence of one agent over another one. Several "germs" (ideas, trends, fashions, ambitions, rules, etc.) can be initiated by one or more agents and eventually adopted by the system. The mechanism defining how these germs are propagated within the network, from the influence of a small set of initially infected nodes, is called a model for influence spread.

Motivated by viral marketing and other applications the problem that has been usually studied is the influence maximization problem initially introduced by Domingos and Richardson [18, 49] and further developed in [39, 21]. This problem addresses the question of finding a set with at most $k$ players having maximum influence, and it is NPhard [18], unless additional restrictions are considered, in which case some generality of the problem is lost [49]. Two

\footnotetext{
This work is partially supported by grant 2009SGR1137 (ALBCOM) of "Generalitat de Catalunya".

Email addresses: xavier.molinero@upc.edu (Xavier Molinero), farisori@lsi.upc.edu (Fabián Riquelme), mjserna@lsi.upc.edu (Maria Serna)

${ }^{1}$ Partially funded by grant MTM2012-34426 of the "Spanish Economy and Competitiveness Ministry".

${ }^{2}$ Supported by grant BecasChile of the "National Commission for Scientific and Technological Research of Chile" (CONICYT).

${ }^{3}$ Supported by grant TIN2013-46181-C2-1-R (COMMAS) of the "Ministerio de Economía y Competitividad".
} 
general models for spread of influence were defined in [39]: the linear threshold model, suggested by [33, 51], and the independent cascade model, created in the context of marketing by [31,32]. Models for influence spread in the presence of multiple competing products have also been proposed and analyzed [9, 12, 2]. In this setting there is also work done towards analyzing the problem from the point of view of non-cooperative game theory. Non-cooperative influence games were defined in 2011 by Irfan and Ortiz [36]. Those games, however, analyze the strategic behavior of two firms competing on the social network and differ from our proposal.

We propose to analyze cooperation in multiagent systems based on a model for influence among the agents in their established network of trust and influence. Social influence is relevant to determine the global behavior of a social network and thus it can be used to enforce cooperation by targeting an adequate initial set of agents. From this point of view we consider a simple and altruistic multiagent system in which the agents are eager to perform a collective task but where their real engagement depends on the perception of the willingness to perform the task of other influential agents. We model the scenario by an influence game, a cooperative simple game in which a team of players (or coalition) succeeds if it is able to convince sufficiently many agents to participate in the task. We take the deterministic linear threshold model $[14,2]$ as the mechanism for influence spread in the associated social network.

In the considered scenario we adopt the natural point of view of decision or voting systems, mathematically modeled as simple games [54]. Simple games were first introduced in 1944 by von Neumann and Morgenstern [54] as a fundamental model for social choice. This point of view brings into the analysis several parameters and properties that are relevant in the study of simple games and thus in the analysis of the proposed scenario. Among those we consider the length and the width, two fundamental parameters that are indicators of efficiency for making a decision [48], or the Shapley-Shubik value (SSval) and the Banzhaf value (Bval), which provide a measure of individual influence. The properties defining proper, strong and decisive games have been considered in the context of simple game theory from its origins [53] and they are also studied. Besides those properties we also consider equivalence and isomorphism. Together with properties of the games there are several properties associated to players that are of interest. Among others we consider the critical players which were used at least since 1965 by Banzhaf [7]. We refer the reader to [53] for a more detailed motivation of the viewpoint of simple games and to [5, 13] for computational aspects of simple games and in general of cooperative game theory.

To define an influence game we take the spread of influence, in the linear threshold model, as the value that measures the power of a team. An influence game is described by an influence graph, modeling a social network, and a quota, indicating the required minimum number of agents that have to cooperate to perform the task successfully. Therefore, a team will be successful, or winning, if it can influence at least as many individuals as the quota requires. Such an approach reveals the importance of the influence between some players over others in order to form successful teams. In this first analysis, we draw upon the deterministic version of the linear threshold model, in which node thresholds are fixed, as our model for influence spread following [14, 2]. It will be of interest to analyze influence games under other spreading models, in particular in the linear threshold model with random thresholds.

Our first result concerns the expressiveness of the family of influence games. We show that unweighted influence games capture the complete family of simple games. Although the construction can be computed in polynomial time when the simple game is given in extensive winning or minimal winning form, the number of winning or minimal winning coalitions is, in general, exponential in the number of players. Interestingly enough the formalization as weighted influence games allows a polynomial time implementation of the operations of intersection and union of weighted simple games, thus showing that, in several cases, simple games that do not admit a succinct representation as weighted games can be represented succinctly as influence games, because their (co)dimension is small.

Our second set of results settles the complexity of problems related to parameters and properties. Hardness results are obtained for unweighted influence games in which the number of agents in the network is polynomial in the number of players, while polynomial time algorithms are devised for general influence games. The new results are summarized in Table 1 as well as the known ones.

We refer the reader to Sections 2 and 4 for a formal definition of all the representations mentioned in the first row and the problems in the first column of Table 1. There P (polynomial time solvable), \#PC (\#P-complete), NPH (NP-hard), coNPH (coNP-hard), coNPC (coNP-complete), QP (quasi-polynomial time solvable) and GIso (the class of problems reducible to graph isomorphism) are known computational complexity classes [29, 44]. The isomorphism problems for simple games, given either by $(N, \mathcal{W})$ or $\left(N, \mathcal{W}^{m}\right)$, are easily shown to be polynomially reducible to the graph isomorphism problem. For games given by $\left(N, W^{m}\right)$, the Iso problem and the graph isomorphism problem are equivalent using arguments from [41]. 


\begin{tabular}{|c|c|c|c|c|c|}
\hline & $\begin{array}{c}\text { Extensive } \\
\text { winning } \\
(N, \mathcal{W})\end{array}$ & $\begin{array}{l}\text { Minimal } \\
\text { winning } \\
\left(N, \mathcal{W}^{m}\right)\end{array}$ & $\begin{array}{c}\text { Weighted } \\
\text { Games } \\
{\left[q ; w_{1}, \ldots, w_{n}\right]}\end{array}$ & \begin{tabular}{|c|}
$\begin{array}{c}\text { Multiple } \\
\text { weighted } \\
\text { games }\end{array}$ \\
\end{tabular} & $\begin{array}{c}\text { Influence } \\
\text { games } \\
(G, w, f, q, N)\end{array}$ \\
\hline LENGTH & $\mathrm{P}$ & $\mathrm{P}$ & $\mathrm{P}[5]$ & NPH[5] & NPH \\
\hline WIDTH & $\mathbf{P}$ & coNPH & $\mathrm{P}[5]$ & $\mathrm{P}[5]$ & NPH \\
\hline BVAL & $\mathrm{P}[4]$ & \#PC[4] & \#PC[16] & \#PC & \#PC \\
\hline SSVAL & $\mathrm{P}[4]$ & \#PC[4] & \#PC[42] & \#PC & \#PC \\
\hline IsDummY & $\mathrm{P}$ & $\mathrm{P}$ & coNPC[42] & coNPC[42] & coNPC \\
\hline ISPASSER & $\mathrm{P}[4]$ & $P[4]$ & $\mathrm{P}[4]$ & $\mathrm{P}[4]$ & $\mathbf{P}$ \\
\hline IsVetoer & $\mathrm{P}[4]$ & $\mathrm{P}[4]$ & $\mathrm{P}[4]$ & $\mathrm{P}[4]$ & $\mathbf{P}$ \\
\hline IsDictator & $\mathrm{P}[4]$ & $\mathrm{P}[4]$ & $\mathrm{P}[4]$ & $\mathrm{P}[4]$ & $\mathbf{P}$ \\
\hline ARESYMMETRIC & $\mathrm{P}$ & $\mathrm{P}$ & coNPC[42] & coNPC[42] & coNPC \\
\hline IsCRITICAL & $P$ & $P$ & $\mathrm{P}$ & $P$ & $\mathbf{P}$ \\
\hline IsBlockING & $\mathrm{P}$ & $\mathrm{P}$ & $\mathrm{P}$ & $\mathrm{P}$ & $\mathbf{P}$ \\
\hline ISPROPER & $\mathrm{P}$ & $\mathrm{P}$ & coNPC[27] & coNPC & coNPC \\
\hline IsSTRONG & $\mathrm{P}$ & coNPC[47] & coNPC[27] & coNPC & coNPC \\
\hline IsDecisive & $\mathrm{P}$ & QP[23] & coNPC[5] & coNPC & coNPC \\
\hline \begin{tabular}{|l} 
EquIV \\
Iso
\end{tabular} & $\begin{array}{c}\mathrm{P} \\
\text { GIso }\end{array}$ & $\begin{array}{c}\mathrm{P} \\
\text { GIso }\end{array}$ & $\begin{array}{c}\text { coNPC }[20] \\
?\end{array}$ & $\begin{array}{c}\text { coNPH }[20] \\
?\end{array}$ & $\begin{array}{l}\text { coNPH } \\
\text { coNPH }\end{array}$ \\
\hline
\end{tabular}

Table 1: Summary of new results (in bold face), known results and trivial results (without reference).

Finally, we consider two extreme cases of influence spread in social networks for undirected and unweighted influence games. In a maximum influence requirement, agents adopt a behavior only when all their peers have already adopted it. This is opposed to a minimum influence requirement in which an agent gets convinced when at least one of its peers does. We show that, in both cases, the problems IsProper, IsStrong and IsDecisive, as well as computing WIDTH, have polynomial time algorithms. Computing LENGTH is NP-hard for maximum influence and polynomial time solvable for minimum influence. For the case of maximum influence and maximum spread, or minimum influence we can show that the problems IsDummy and AreSymmetric belong to P.

\section{Definitions and Preliminaries}

Before introducing formally the family of influence games we need to define a family of labeled graphs and a process of spread of influence based on the linear threshold model [33,51]. We use standard graph notation following [10]. As usual, given a finite set $U, \mathcal{P}(U)$ denotes its power set, and $|U|$ its cardinality. For any $0 \leq k \leq|U|, \mathcal{P}_{k}(U)$ denotes the subsets of $U$ with exactly $k$ elements. For a given graph $G=(V, E)$ we assume that $n=|V|$ and $m=|E|$. Also $G[S]$ denotes the subgraph induced by $S \subseteq V$ and, for a vertex $u \in V, N(u)=\{v \in V \mid(u, v) \in E\}$.

Definition 1. An influence graph is a tuple $(G, w, f)$, where $G=(V, E)$ is a weighted, labeled and directed graph (without loops). As usual $V$ is the set of vertices or agents, $E$ is the set of edges and $w: E \rightarrow \mathbb{N}$ is a weight function. Finally, $f: V \rightarrow \mathbb{N}$ is a labeling function that quantifies how influenceable each agent is. An agent $i \in V$ has influence over another agent $j \in V$ if and only if $(i, j) \in E$. We also consider the family of unweighted influence graphs $(G, f)$ in which every edge has weight 1.

Given an influence graph $(G, w, f)$ and an initial activation set $X \subseteq V$, the spread of influence of $X$ is the set $F(X) \subseteq V$ which is formed by the agents activated through an iterative process. We use $F_{k}(X)$ to denote the set of nodes activated at step $k$. Initially, at step 0 , only the vertices in $X$ are activated, that is $F_{0}(X)=X$. The set of vertices activated at step $i>0$ consists of all vertices for which the total weight of the edges connecting them to nodes in $F_{i-1}(X)$ meets or exceeds their labels, i.e.,

$$
F_{i}(X)=F_{i-1}(X) \cup\left\{v \in V \mid \sum_{\left\{u \in F_{i-1}(X) \mid(u, v) \in E\right\}} w((u, v)) \geq f(v)\right\} .
$$



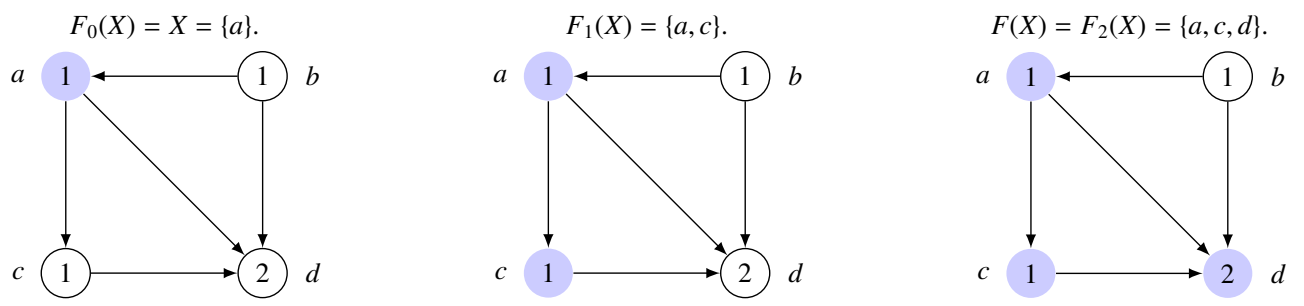

Figure 1: The spread of influence starting from the initial activation of $X=\{a\}$ on an unweighted graph.

The process stops when no additional activation occurs and the final set of activated nodes is denoted by $F(X)$.

Example 1. Figure 1 shows the spread of influence $F(X)$ in an unweighted influence graph $G=(V, f)$, with $V=$ $\{a, b, c, d\}$, for the initial activation $X=\{a\}$. In the first step we obtain $F_{1}(X)=\{a, c\}$, and in the second step (the last one) we obtain $F(X)=F_{2}(x)=\{a, c, d\}$.

As the number of vertices is finite, for any $i>n, F_{i}(X)=F_{i-1}(X)$. Thus, $F(X)=F_{n}(X)$ and we have the following well known basic result.

Lemma 1. Given an influence graph $(G, w, f)$ and a set of vertices $X$, the set $F(X)$ can be computed in polynomial time.

In what follows, unless otherwise stated, results and definitions will be stated for directed graphs. All of them can be restated for undirected graphs. Now we define influence games.

Definition 2. An influence game is given by a tuple $(G, w, f, q, N)$ where $(G, w, f)$ is an influence graph, $q$ is an integer quota, $0 \leq q \leq|V|+1$, and $N \subseteq V$ is the set of players. $X \subseteq N$ is a successful team if and only if $|F(X)| \geq q$, otherwise $X$ is an unsuccessful team.

As it was done for influence graphs, we also consider the family of unweighted influence games for the cases in which the graph $G$ is unweighted. In such a case we use the notation $(G, f, q, N)$.

An influence game corresponds to a mathematical model called simple game, where teams are coalitions, and agents act as players. Simple games were first introduced in 1944 by Neumann and Morgenstern [54], but using the definition that corresponds to the so called strong games, which will be defined later. The first definition that captures the current understanding of simple games was given in 1953 by Gillies [30]. In this scenario of simple games, we follow definitions and notation from [53]. A family of subsets $\mathcal{W} \subseteq \mathcal{P}(N)$ is said to be monotonic when, for any $X \in \mathcal{W}$ and $Z \in \mathcal{P}(N)$, if $X \subseteq Z$, then $Z \in \mathcal{W}$.

Definition 3. A simple game $\Gamma$ is given by a tuple $(N, \mathcal{W})$ where $N$ is a finite set of players and $\mathcal{W}$ is a monotonic family of subsets of $N$.

In the context of simple games, the subsets of $N$ are called coalitions, $N$ is the grand coalition and $X \in \mathcal{W}$ is a winning coalition (a successful team). Any subset of $N$ which is not a winning coalition is called a losing coalition (an unsuccessful team). A minimal winning coalition is a winning coalition $X$ that does not properly contain any winning coalition. That is, removing any player from $X$ results in a losing coalition. A maximal losing coalition is a losing coalition $X$ that is not properly contained in any other losing coalition. That is, adding any player to $X$ results in a winning coalition. We use $\mathcal{W}, \mathcal{L}, \mathcal{W}^{m}$ and $\mathcal{L}^{M}$ to denote the sets of winning, losing, minimal winning and maximal losing coalitions, respectively. Any of those set families determine uniquely the game and constitute the usual forms of representation for simple games [53], although the sizes of those representations are not, in general, polynomial in the number of players. 
Example 2. Let $(G, f)$ be an influence graph and $N$ any subset of agents. Two particular ranges of the quota lead to some trivial simple games. By setting $q=0$, thus considering influence games of the form $(G, f, 0, N)$, we have that every team of agents is successful, therefore $(G, f, 0, N)$ is a representation of the simple game $(N, \mathcal{P}(N))$. When $q>|V(G)|$, the influence game $(G, f, q, N)$ is a representation of the simple game $(N, \emptyset)$ as there are no successful teams in the game.

Let us provide an example of an influence game based on the influence graph considered in Example 1.

Example 3. Consider the influence game $\Gamma=(G, f, 3, V(G))$, where $(G, f)$ is the influence graph considered in Example 1. In this case, we have that $F(\{a\})=\{a, c, d\}$, and thus $\{a\} \in \mathcal{W}$. The fundamental set families for $\Gamma$ are:

$$
\mathcal{W}^{m}=\{\{a\},\{b\}\} \quad \mathcal{L}^{M}=\{\{c, d\}\} \quad \mathcal{L}=\{\{c, d\},\{c\},\{d\},\{\}\} \quad \mathcal{W}=\mathcal{P}(V(G)) \backslash \mathcal{L} .
$$

The intersection of two simple games is the simple game where a coalition wins if and only if it wins in both games. In a similar way, the union of two simple games is the simple game where a coalition wins if and only if it wins in at least one of the two games [53].

Finally, we introduce a subfamily of simple games, the weighted games.

Definition 4. A simple game $(N, \mathcal{W})$ is a weighted game (also called a weighted voting game) if there exists a weight function $w: N \rightarrow \mathbb{R}^{+}$and a quota $q \in \mathbb{R}$, such that, for any $X \subseteq N, X \in \mathcal{W}$ if and only if $w(X) \geq q$, where $w(X)=\sum_{i \in X} w(i)$.

A weighted game can be represented by a weighted representation, i.e., a vector $\left[q ; w_{1}, \ldots, w_{n}\right]$ where $w_{i}=w(i)$, for any $i \in N$, and $q \in \mathbb{R}$ is a quota, defining the simple game in which $S \in \mathcal{W}$ if and only if $w(S) \geq q$. According to $\mathrm{Hu}$ [35] (see also [26]) the weighted representations can be restricted to integer non-negative weights with $0 \leq q \leq$ $w(N)$. Multiple weighted games are simple games defined by the intersection of a finite collection of weighted games.

Despite the fact that weighted games are a strict subclass of simple games, it is known that every simple game can be expressed as an intersection or a union of a finite number of weighted games. The result for intersection (dimension concept) was first shown in [38] for hypergraphs, and then expressed for simple games in [52]. The result for union (codimension concept) was introduced for simple games in [25]. A simple game is said to be of dimension (codimension) $k$ if and only if it can be represented as the intersection (union) of exactly $k$ weighted games, but not as the intersection (union) of $(k-1)$ weighted games. It is known that given $k$ weighted games, to decide whether the dimension of their intersection exactly equals $k$ is NP-hard [17]. A generalization of games constructed through binary operators is the family of boolean weighted games introduced in [22]. A boolean weighted game is defined by a propositional logic formula and a finite collection of weighted games. The boolean formula determines the requirements for a coalition to be winning in the described game. When considering only monotone formulas, boolean weighted games provide another representation of simple games.

\section{Expressiveness}

Influence games are monotonic as, for any $X \subseteq N$ and $i \in N$, if $|F(X)| \geq q$ then $|F(X \cup\{i\})| \geq q$, and if $|F(X)|<q$ then $|F(X \backslash\{i\})|<q$. Thus, every influence game is a simple game. Moreover, we will show that the opposite is also true. From now on, in the following figures, those nodes that are players are shaded in grey.

Theorem 1. Every simple game can be represented by an unweighted influence game. Furthermore, when the simple game $\Gamma$ is given by either $(N, \mathcal{W})$ or $\left(N, \mathcal{W}^{m}\right)$, an unweighted influence game representing $\Gamma$ can be obtained in polynomial time.

Proof. Assume that a simple game $\Gamma$ is given by $(N, \mathcal{W})$ or $\left(N, \mathcal{W}^{m}\right)$. It is already well known that given $(N, \mathcal{W})$, the family $\mathcal{W}^{m}$ can be obtained in polynomial time. Thus we assume in the following that the set of players and the set $\mathcal{W}^{m}$ are given.

In order to represent $\Gamma$ as an influence game we first define an unweighted influence graph $(G, f)$. The graph $G=(V, E)$ is constructed as follows. The set $V$ of nodes contains $n$ nodes, $V_{N}=\left\{v_{1}, \ldots, v_{n}\right\}$, one for each player, and a set of nodes for each minimal winning coalition. For any $X \in \mathcal{W}^{m}$, we add a new set $V_{X}$ with $n+1-|X|$ nodes. 


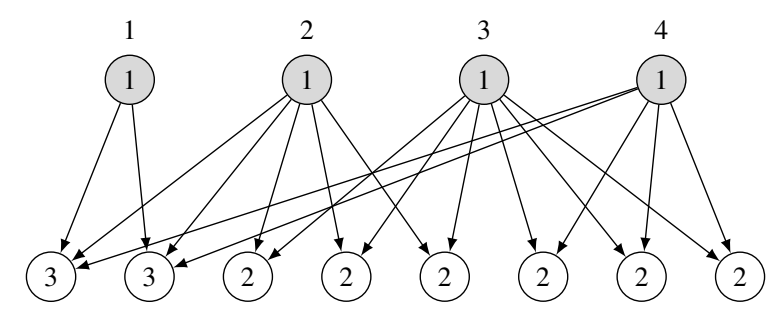

Figure 2: An unweighted influence graph associated to the simple game $(\{1,2,3,4\},\{\{1,2,4\},\{2,3\},\{3,4\}\})$.

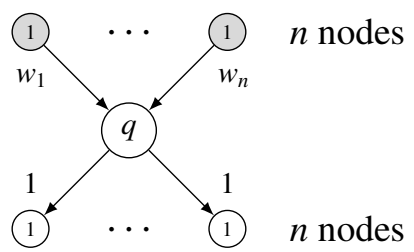

Figure 3: An influence graph $(G, w, f)$ associated to the weighted game $\left[q ; w_{1}, \ldots, w_{n}\right]$.

We connect vertex $v_{i}$ with all the vertices in $V_{X}$ whenever $i \in X$. Finally, the label function is defined as follows: for any $1 \leq i \leq n, f\left(v_{i}\right)=1$ and, for any $X \in \mathcal{W}^{m}$ and any $v \in V_{X}, f(v)=|X|$. Observe that in the influence game $\left(G, f, n+1, V_{N}\right)$ a team is successful if and only if its players form a winning coalition in $\Gamma$. Therefore $\left(G, f, n+1, V_{N}\right)$ is a representation of $\Gamma$ as unweighted influence game.

Given $\left(N, \mathcal{W}^{m}\right)$, since a description of $(G, f, n+1, N)$ requires only a polynomial number of vertices in terms of $n$, then this description can be computed in polynomial time.

The following example provides an illustration of the construction.

Example 4. Let $\Gamma=(\{1,2,3,4\},\{\{1,2,4\},\{2,3\},\{3,4\}\})$ be a simple game in minimal winning form. For coalition $\{1,2,4\}$ we add $n+1-|\{1,2,4\}|=4+1-3=2$ nodes to the graph with label 3 . For each of the coalitions $\{2,3\}$ and $\{3,4\}$ we need to add 3 nodes with label 2. A drawing of the resulting unweighted influence graph is given in Figure 2.

The proof of Theorem 1 shows the expressiveness of the family of influence games with respect to the class of simple games. However, the construction cannot be implemented in polynomial time when the simple game is given in succinct way, e.g., by a weighted representation or as a monotonic Boolean function. Observe also that the number of agents in the corresponding influence game is in general exponential in the number of players. For the particular case of weighted games, with given weighted representation, we can show that there exist representations by influence games having a polynomial number of agents.

Theorem 2. Every weighted game can be represented as an influence game. Furthermore, given a weighted representation of the game, a representation as an influence game can be obtained in polynomial time.

Proof. Let $\left[q ; w_{1}, \ldots, w_{n}\right]$ be a weighted game, and consider the influence game $(G, w, f, n+1, N)$, whose influence graph is shown in Figure 3. The $n$ nodes in the first level of $G$ correspond to the set $N$, each of them has 1 as its associated label. Each node $i \in N$ is connected to a central node with label $q$ and the corresponding edge has weight $w_{i}$. The $n$ nodes in the last level are another set of $n$ nodes with label 1 . Observe that, $X \subseteq N$ is a winning coalition in $\left[q ; w_{1}, \ldots, w_{n}\right]$ if and only if $\sum_{i \in X} w_{i} \geq q$. The last condition is equivalent to $|F(X)| \geq n+1$. Thus we have that $X \subseteq N$ is a winning coalition in $\left[q ; w_{1}, \ldots, w_{n}\right]$ if and only if $X \subseteq N$ is a winning coalition in $(G, w, f, n+1, N)$.

Finally, observe that the construction of $(G, w, f, n+1, N)$ can be constructed in polynomial time with respect to the size of $\left[q ; w_{1}, \ldots, w_{n}\right]$. 


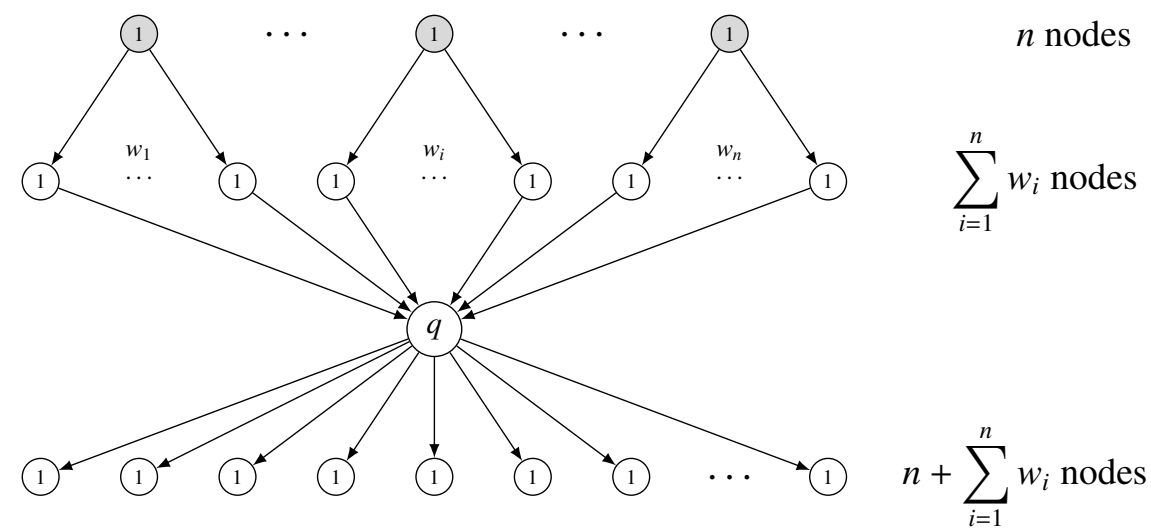

Figure 4: An unweighted influence graph $(G, f)$ associated to the weighted game $\left[q ; w_{1}, \ldots, w_{n}\right]$.

Observe that in the previous construction the size of the influence graph is polynomial in the number of agents but the overall construction is done in polynomial time in the size of the weighted representation. We can slightly change the construction and get a representation as unweighted influence game by increasing again the number of players.

Theorem 3. Every weighted game can be represented as an unweighted influence game. Furthermore, given a weighted representation of the game, a representation as unweighted influence game can be obtained in pseudopolynomial time.

Proof. Let $\left[q ; w_{1}, \ldots, w_{n}\right]$ be a weighted game, and consider the unweighted influence graph $(G, f)$ sketched in Figure 4. The $n$ nodes in the first level correspond to the set $N$. For any $i \in N$, node $i$ is connected to a set of $w_{i}$ different nodes in the second level representing its weight. Thus, $X \subseteq N$ is a winning coalition if and only if $\sum_{i \in X} w_{i} \geq q$, which is equivalent to $|F(X)| \geq n+\sum_{i=1}^{n} w_{i}$. Therefore, the influence game $\left(G, f, n+\sum_{i=1}^{n} w_{i}, N\right)$ is a representation of the given weighted game.

Observe that given $\left[q ; w_{1}, \ldots, w_{n}\right]$, constructing the graph $G$ requires time $O\left(n+w_{1}+\cdots+w_{n}\right)$ and thus the construction can be done in pseudo-polynomial time.

In the previous results we have assumed that a weighted representation of the game is given. It is known that there are weighted games whose weighted representation requires $\max _{i \in N}\left\{w_{i}\right\}$ to be $(n+1)^{(n+1) / 2} / 2$ [45]. Therefore the construction of Theorem 2 will require exponential space and time with respect to the number of players.

Our next result establishes the closure of influence games under intersection and union. Furthermore, we show that an influence game representing the resulting simple game can be obtained in polynomial time.

Theorem 4. Given two influence games, their intersection and union can be represented as an influence game. Furthermore, both constructions can be obtained in polynomial time.

Proof. Let $\Gamma=(G, w, f, q, N)$ be an influence game with $G=(V, E)$, and recall that, for any $X \subseteq N, F_{i}(X) \subseteq V$ denotes the spread of influence of $X$ in the $i$-th step of the activation process and that we can assume that $0 \leq i \leq n$. All the sets considered in our constructions are replications of either the set $N$ or the set $V$. For sake of simplicity, we use the term corresponding node to refer to the same node in a different copy of $N$ or $V$.

We start constructing an influence graph $\left(G^{\prime}, w^{\prime}, f^{\prime}\right)$ as shown in Figure 5. $G^{\prime}$ has $2 n+1$ columns of nodes. The first column $F^{0}$ represents $V$, and the remaining nodes are divided in pairs of sets $\left(f^{i}, F^{i}\right)$, for any $1 \leq i \leq n$. For any $1 \leq i \leq n$, the sets $f^{i}$ and $F^{i}$ have $n$ nodes each, as a replication of the nodes in $V$. The edges are defined as follows: for any $1 \leq i \leq n$, a node $y \in F^{i-1}$ is connected to a node $z \in f^{i}$ if and only if $(y, z) \in E$. These edges have associated weight $w((y, z))$. Furthermore, every node in $F^{i-1}$ is connected by an edge of weight 1 to its corresponding node in $F^{i}$. Every node in $f^{i}$ is connected by an edge of weight 1 to its corresponding node in $F^{i}$. The labeling function assigns label 1 to all the nodes in sets $F^{i}$ and maintains the original labeling for nodes in the sets $f^{i}$. 


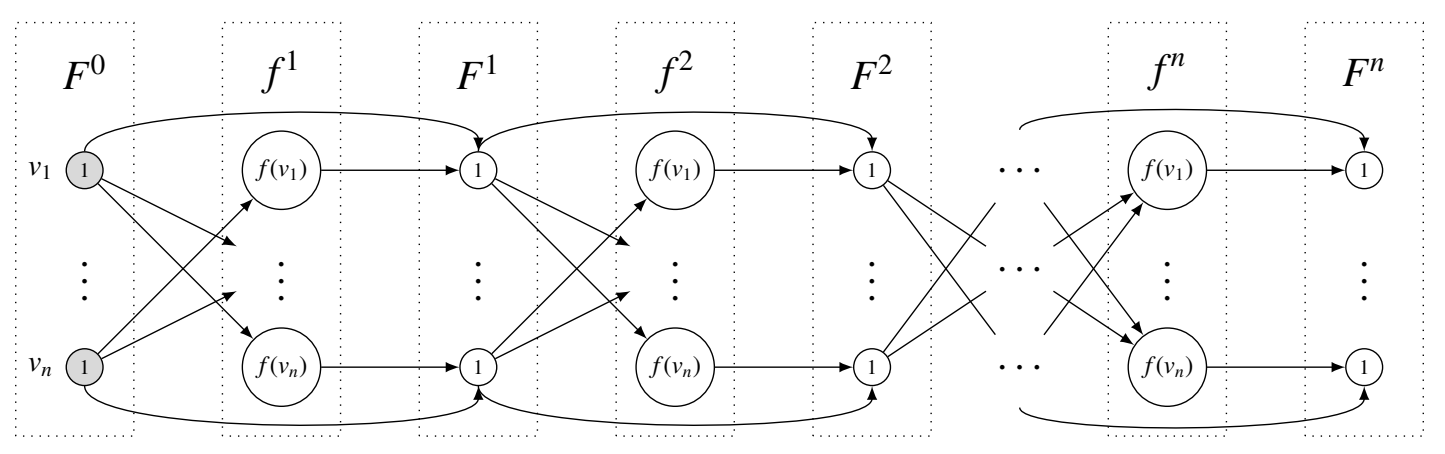

Figure 5: The influence graph $\left(G^{\prime}, w^{\prime}, f^{\prime}\right)$ associated to the influence game $(G, w, f, q, N)$.

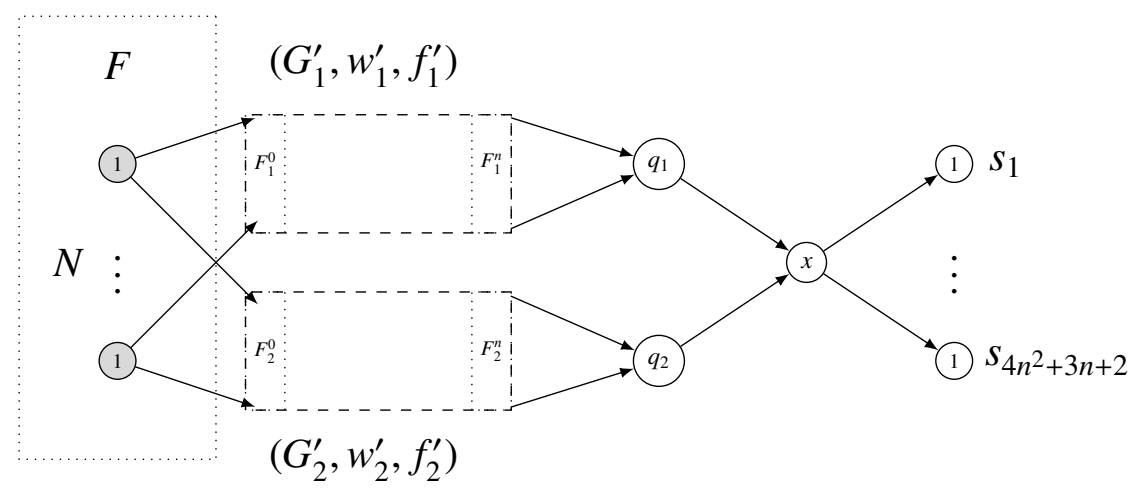

Figure 6: The influence graph associated to the intersection $(x=2)$ or the union $(x=1)$ of two influence games with influence graphs $\left(G_{1}, w_{1}, f_{1}\right)$ and $\left(G_{2}, w_{2}, f_{2}\right)$ and quotas $q_{1}$ and $q_{2}$ respectively.

Note that after the activation of a team $X \subseteq F^{0}$ in $\left(G^{\prime}, w^{\prime}, f^{\prime}\right)$, for any $0 \leq i \leq n$, the set of nodes in $F^{i}$ that are activated coincides with the set $F_{i}(X)$. Thus the subset of activated nodes in $F^{n}$ coincides with $F(X)$. Observe also that $\left(G^{\prime}, w^{\prime}, f^{\prime}\right)$ has $2 n^{2}+n$ nodes and that it can be constructed in polynomial time in the size of a given influence game $(G, w, f, q, N)$.

Now, given two influence games $\Gamma_{1}=\left(G_{1}, w_{1}, f_{1}, q_{1}, N\right)$ and $\Gamma_{2}=\left(G_{2}, w_{2}, f_{2}, q_{2}, N\right)$ we construct the two influence graphs $\left(G_{1}^{\prime}, w_{1}^{\prime}, f_{1}^{\prime}\right)$ and $\left(G_{2}^{\prime}, w_{2}^{\prime}, f_{2}^{\prime}\right)$ as described before (see Figure 5). We use the construction depicted in Figure 6 to construct another influence graph. In this last construction we add a set $F$ which is a copy of $N$. All the nodes in $F$ have label 1. The nodes in $F$ are connected to their corresponding nodes in $F_{1}^{0}$ and in $F_{2}^{0}$ through edges of weight 1. Furthermore, we add a node with label $q_{1}$, a node with label $q_{2}$, a node with label $x$, and a set with $4 n^{2}+3 n+2$ nodes. Those new nodes are connected according to the pattern given in Figure 6 . The nodes in the last column, $F_{i}^{n}$, of $\left(G_{i}^{\prime}, w_{i}^{\prime}, f_{i}^{\prime}\right)$ are all connected to the node with label $q_{i}$, for $i \in\{1,2\}$. The nodes with labels $q_{1}$ and $q_{2}$ are connected to the node with label $x$ which is connected to the last set of nodes. All those new connections have weight 1 . Observe that in total we have at most $2\left(2 n^{2}+n\right)+n+3+4 n^{2}+3 n+2$ nodes. Thus the overall construction can be computed in polynomial time.

Let $\left(G_{\cup}, w_{\cup}, f_{\cup}\right)$ be the influence graph obtained by setting $x=1$ and $\left(G_{\cap}, w_{\cap}, f_{\cap}\right)$ be the influence graph obtained by setting $x=2$. Consider the games $\Gamma_{\cup}=\left(G_{\cup}, w_{\cup}, f_{\cup}, 4 n^{2}+3 n+2, F\right)$ and $\Gamma_{\cap}=\left(G_{\cap}, w_{\cap}, f_{\cap}, 4 n^{2}+3 n+2, F\right)$. By construction a team $X$ is successful in $\Gamma_{\cup}$ if and only if $X$ is successful in either $\Gamma_{1}$ or $\Gamma_{2}$. Furthermore, a team is successful in $\Gamma_{\cap}$ if and only if $X$ is successful in both $\Gamma_{1}$ and $\Gamma_{2}$.

It is interesting to note that it is possible to devise a construction representing the intersection or the union of 


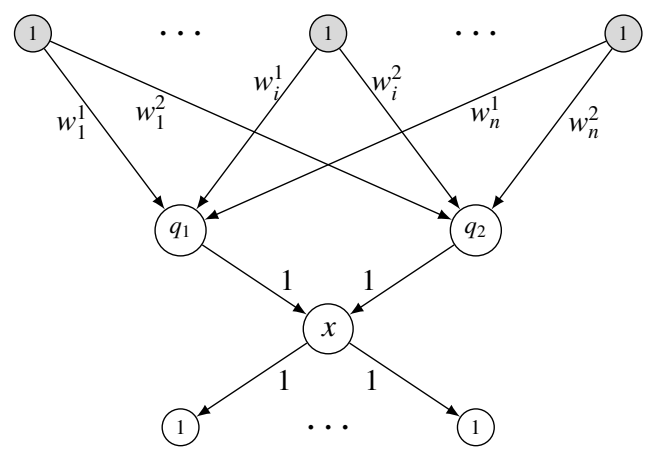

$n$ nodes

$n$ nodes

Figure 7: Influence graphs associated to $\left[q_{1} ; w_{1}^{1}, \ldots, w_{n}^{1}\right] \cap\left[q_{2} ; w_{1}^{2}, \ldots, w_{n}^{2}\right](x=2)$, and $\left[q_{1} ; w_{1}^{1}, \ldots, w_{n}^{1}\right] \cup\left[q_{2} ; w_{1}^{2}, \ldots, w_{n}^{2}\right](x=1)$.

weighted games as the influence games $\left(G_{\cup}^{\prime}, w^{\prime}, f_{\cup}^{\prime}, n+2, N\right)$ and $\left(G_{\cap}^{\prime}, w^{\prime}, f_{\cap}^{\prime}, n+3, N\right)$. The corresponding influence graphs $\left(G_{\cup}^{\prime}, w^{\prime}, f_{\cup}^{\prime}\right)$ and $\left(G_{\cap}^{\prime}, w^{\prime}, f_{\cap}^{\prime}\right)$ are shown in Figure 7 (setting as before label $x$ to be 1 or 2 depending on the considered operation). This new construction requires only a linear number of additional nodes, and the graph is weighted.

Thus, as any simple game can be represented as an intersection or union of a finite number of weighted games, we have an alternative way to show the completeness of the family of weighted influence games with respect to the class of simple games (Theorem 1). However, as the dimension, the codimension, and the representation as a boolean weighted game of a simple game might be exponential in the number of players (but bounded by the number of maximal losing, minimal winning coalitions, or both, respectively) [28, 25, 22], we cannot conclude that any simple game can be represented by a weighted influence game whose number of agents is polynomial in the number of players. For the particular case of unweighted influence game we know the following.

Theorem 5. The family of unweighted influence games in which the number of agents in the corresponding influence graph is polynomial in the number of players is a proper subset of simple games.

Proof. We use a simple counting argument to show the result. Observe that, for any $n \geq 0$, there are more than $2^{\left(2^{n} / n\right)}$ simple games with $n$ players [40]. Taking into account that a simple game has at most $n$ ! isomorphic simple games we know that there are more that $2^{\left(2^{n} / n\right)} / n$ ! different simple games on $n$ players.

Consider an unweighted influence game with $n$ players and $f(n)$ agents. The possibilities for the edge sets are less than $2^{(f(n)+1)^{2}}$. It suffices to consider label functions assigning values between 0 and $f(n)+1$. Thus, there are at most $(f(n)+2)^{f(n)+2}$ possibilities for the labeling functions. Finally, for the quota, only $f(n)+2$ possibilities have to be considered. Thus, the number of unweighted influence games with $n$ players and $f(n)$ agents is at most $2^{O\left(f(n)^{2}\right)}$.

Taking $f(n)=n^{\log n}$, the family includes all unweighted influence games with $n$ players and polynomial number of agents. Taking the logarithm on both sides, one easily sees that $2^{O\left(f(n)^{2}\right)}$ is asymptotically smaller than $2^{\left(2^{n} / n\right)} / n$ !.

\section{Parameters and Properties}

From Theorems 1 and 2 we know that all the computational problems related to properties and parameters that are computationally hard for simple games in winning or minimal winning form, as well as for weighted games, are also computationally hard for influence games. Nevertheless, the hardness results do not apply to unweighted influence games with polynomial, in the number of players, number of agents. In this section we address the computational complexity of problems for games with a polynomial number of agents. All the hardness proofs are given for the subclass formed by unweighted influence games on undirected influence graphs, which is a subset of all the other variations. The polynomial time algorithms are devised for the biggest class of general influence games, i.e., weighted influence games on directed graphs which includes all others.

Before starting to analyze problems we state here some results using Lemma 1. 
Theorem 6. For a given influence game $(G, w, f, q, N)$, deciding whether a team $X \subseteq N$ is successful can be done in polynomial time.

Our next result concerns a particular type of influence games that we will use first as a basic construction, which associates an unweighted influence game to an undirected graph, and later as a representative of a particular subclass of influence games.

Definition 5. Given an undirected graph $G=(V, E)$, the unweighted influence game $\Gamma(G)$ is the game $(G, f,|V|, V)$ where, for any $v \in V$, the label $f(v)$ is the degree of $v$ in $G$, i.e., $f(v)=d_{G}(v)$.

Recall that a set $S \subseteq V$ is a vertex cover of a graph $G$ if and only if, for any edge $(u, v) \in E, u$ or $v$ (or both) belong to $S$. From the definitions we get the following result.

Lemma 2. Let $G$ be an undirected graph. A team $X$ is successful in $\Gamma(G)$ if and only if $X$ is a vertex cover of $G$, Furthermore, the influence game $\Gamma(G)$ can be obtained in polynomial time, given a description of $G$.

Table 1 in Section 1 summarizes the known computational complexity results (notation from [29]) over parameters and properties [16, 23, 42, 46, 5, 27, 50]. For the hardness results we provide polynomial time reductions from the following problems (decision or optimization versions) which are known to be NP-hard [29].

Vertex Cover: Given an undirected graph $G=(V, E)$ and an integer $k$.

Does $G$ have a vertex cover with size $k$ or less?

Hitting Set: $\quad$ Given a collection $C$ of subsets of a finite set $S$, and an integer $k$.

Is there a subset $S^{\prime} \subseteq S$ with $\left|S^{\prime}\right| \leq k$ such that

for every subset $C^{\prime} \in C$ we have $S^{\prime} \cap C^{\prime} \neq \emptyset$ ?

SET Cover: $\quad$ Given a collection $C$ of subsets of a finite set $S$, and an integer $k$.

Is there a collection of sets $C^{\prime} \subseteq C$ with $\left|C^{\prime}\right| \leq k$ such that every element in $S$ belongs to at least one member of $C^{\prime}$ ?

Set Packing: $\quad$ Given a collection $C$ of finite sets, and an integer $k$.

Is there a collection of disjoint sets $C^{\prime} \subseteq C$ with $\left|C^{\prime}\right| \geq k$ ?

We first analyze the complexity of two relevant simple game measures that provide information about the size of the teams that is sufficient/necessary to succeed. Further, we consider two new measures which are relevant for influence games: the strict length, defined in Section 3, and its dual, the strict width. Assume that a simple game $\Gamma$ is given.

LeNGTH: $\quad$ The minimum size of a successful team, i.e., $\min \{|X| \mid X \in \mathcal{W}\}$.

WIDTH: $\quad$ The maximum size of an unsuccessful team, i.e., $\max \{|X| \mid X \in \mathcal{L}\}$.

SLENGTH: $\quad$ The minimum cardinality from which all teams are successful, i.e., $\min \left\{k \in \mathbb{N} \mid \mathcal{P}_{k}(N) \subseteq \mathcal{W}\right\}$.

sWIDTH: $\quad$ The maximum cardinality to which all teams are unseccessful, i.e., $\max \left\{k \in \mathbb{N} \mid \mathcal{P}_{k}(N) \subseteq \mathcal{L}\right\}$.

Observe that, for every simple game $\Gamma, \operatorname{Width}(\Gamma)=\operatorname{sLength}(\Gamma)-1$ and $\operatorname{Length}(\Gamma)=\operatorname{sWidth}(\Gamma)+1$.

Before study the complexity of the parameters in the context of influence games, we states their complexity for simple games given by $(N, \mathcal{W})$ and $\left(N, \mathcal{W}^{m}\right)$. It is well known that under these forms of representation, LeNGTH can trivially be computed in polynomial time. As $\operatorname{LENGTH}(\Gamma)=\operatorname{sWIDTH}(\Gamma)+1$, $\mathrm{sW}$ IDTH can also be computed in polynomial time as well. However, the WIDTH problem presents more difficulties. Computing WIDTH for simple games given by $(N, \mathcal{W})$ or $\left(N, \mathcal{W}^{m}\right)$ was posted as an open problem in [5]. Now we prove that this problem is hard when the game is given by $\left(N, \mathcal{W}^{m}\right)$. The proof is based in the computation of the sLENGTH parameter. 
Theorem 7. Given a simple game by $(N, \mathcal{W})$, WIDTH and SLENGTH can be computed in polynomial time. When the game is given by $\left(N, W^{m}\right)$, they become coNP-hard.

Proof. Given $(N, \mathcal{W})$, it is clear that sLength can be computed in polynomial time. Since $\operatorname{Width}(\Gamma)=\operatorname{sLeNGTH}(\Gamma)-1$, then WIDTH can also be computed in polynomial time.

Given $\left(N, W^{m}\right)$, to prove coNP-hardness we provide a reduction from the HitTing SET problem given by an instance $(S, C, k)$ (see the definition above). Observe that we can assume that no set in $C$ is a subset of another set in $C$ : if $C_{1}^{\prime} \subset C_{2}^{\prime}$ for some $C_{1}^{\prime}, C_{2}^{\prime} \in C$, we can remove $C_{2}^{\prime}$ from $C$ without changing the answer. Under this assumption, an intance $(S, C, k)$ of Hitring Set corresponds to a simple game $\Gamma$ that has $S$ as its set of players and $C$ as its list of minimal winning coalitions. By construction, $S^{\prime}$ is a hitting set for $C$ if and only if $S \backslash S^{\prime}$ is a losing coalition in $\Gamma$. Therefore, $C$ admits a hitting set of size at most $k$ if and only if $\Gamma$ has a losing coalition of size at least $|S|-k$, i.e., if $\operatorname{sLength}(\Gamma)>|S|-k$. This completes the reduction.

Our next result shows that computing the four measures defined above is hard for influence games.

Theorem 8. Computing Length, WIDTh, SLEngth and SWIDTh of an unweighted influence game is NP-hard.

Proof. For the measure Length, we provide a reduction from the MINIMUM SET Covering problem. Let $C=\left\{C_{1}, \ldots, C_{m}\right\}$ be a collection of subsets of a universe with $n$ elements. We associate to $C$ the unweighted influence game $(G, f, q, N)$ where $G=(V, E)$. The graph $G$ has three disjoint sets of vertices: $Y=\left\{y_{1}, \ldots, y_{m}\right\}, T=\left\{t_{1}, \ldots, t_{n}\right\}$, and $Z=$ $\left\{z_{1}, \ldots, z_{m+1}\right\}$, together with an additional vertex $x$. The components of the game are the following.

- $V=Y \cup T \cup\{x\} \cup Z$,

- $E=\left\{\left(y_{j}, t_{i}\right) \mid i \in C_{j}\right\} \cup\left\{\left(t_{i}, x\right) \mid 1 \leq i \leq n\right\} \cup\left\{\left(x, z_{k}\right) \mid 1 \leq k \leq m+1\right\}$,

- $f\left(y_{j}\right)=n+1$, for any $1 \leq j \leq m$,

- $f\left(t_{i}\right)=1$, for any $1 \leq i \leq n$,

- $f\left(z_{k}\right)=1$, for any $1 \leq k \leq m+1$,

- $f(x)=n$,

- $q=m+n+1$ and

- $N=Y$.

Therefore, it is easy to see that a team $X \subseteq N$ succeeds if and only if it corresponds to a set cover, so the LENGTH of $(G, f, q, N)$ coincides with the size of a minimum set cover.

For the measure WIDTH we provide a reduction from the MAXIMUM SET PACKING problem. Consider an influence game $\left(G^{\prime}, f^{\prime}, q^{\prime}, N\right)$ where $G^{\prime}$ is constructed from $G$. We remove node $\{x\}$, add the connections $\left\{\left(t_{i}, z_{k}\right) \mid 1 \leq i \leq n, 1 \leq\right.$ $k \leq m+1\}$, and set $f^{\prime}\left(t_{i}\right)=2$ for any $1 \leq i \leq n$. We keep $N=Y$ and set $q^{\prime}=m+1$. It is easy to see that a team $X \subseteq N$ is unsuccessful in $\left(G^{\prime}, f^{\prime}, q^{\prime}, N\right)$ if and only if $X$ corresponds to a set packing in $C$. Therefore, the WIDTH of $\left(G^{\prime}, f^{\prime}, q^{\prime}, N\right)$ is the size of a maximum set packing of $C$.

The remaining results for sLENGTH and sWIDTH follow from the relationships $\operatorname{WidTH}(\Gamma)=\operatorname{sLENGTH}(\Gamma)-1$ and $\operatorname{LENGTH}(\Gamma)=\operatorname{sWIDTH}(\Gamma)+1$.

The hardness result for LENGTH can be obtained directly from Lemma 2. There we provide a reduction from the MINIMUM VERTEX COVER problem. However, the reduction from the MINIMUM SET COVERING problem given in the previous theorem allows us to extract additional results about the complexity of approximation. In particular, the reductions in Theorem 8 imply that Length is neither approximable within $(1-\epsilon) \cdot \log m$ nor within $c \cdot \log n$, for some $c>0$, and that WIDTH is not approximable within $m^{1 / 2-\epsilon}$, for any $\epsilon>0$, using the non-approximability results from [3] for the MAXIMUM SET PACKING and MINIMUM SET COVERING problems.

Our next result settles the complexity of the computation of the Banzhaf and Shapley-Shubik values of a given player. 
Definition 6 ([24]). Let $\Gamma=(N, \mathcal{W})$ be a simple (influence) game. For any $i \in N$, let $C_{i}=\{S \in \mathcal{W} ; S \backslash\{i\} \notin \mathcal{W}\}$ be the set of blocking teams (coalitions) for player $i$. The Banzhaf value of $i$ on $\Gamma$ is $\eta_{i}(\Gamma)=\left|C_{i}\right|$ and the Shapley-Shubik value of $i$ on $\Gamma$ is

$$
\kappa_{i}(\Gamma)=\sum_{S \in C_{i}}(|S|-1) !(n-|S|) !
$$

The Banzhaf index of $i$ on $\Gamma$ is $\eta_{i}(\Gamma) / 2^{n-1}$ and the Shapley-Shubik index of $i$ on $\Gamma$ is $\kappa_{i}(\Gamma) / n !$.

The computational complexity of the two indices is the same as that of the corresponding values. We analyze here only the problems of computing the values. The two problems are denoted as BVAL and SSvaL, respectively.

Theorem 9. Computing BvaL and SSvaL for a given influence game and a given player is \#P-complete.

Proof. Both problems belong trivially to \#P. To show hardness we construct a reduction from the problem of computing the number of vertex covers of a given graph, which is known to be \#P-complete [29]. Given a graph $G$, we first construct the graph $G^{\prime}$ which is obtained from $G$ by adding a new vertex $x$ and connecting $x$ to all the vertices in $G$. The associated input to BvaL is formed by the influence game $\Gamma\left(G^{\prime}\right)$ and the player $x$. Observe that the reduction can be computed in polynomial time.

Let $X$ be a successful team in $\Gamma\left(G^{\prime}\right)$ such that $x \in X$. When $X \neq V\left(G^{\prime}\right)$ we know that $X \backslash\{x\}$ must be a vertex cover of $G$. Furthermore $X \in C_{x}$ as $X \backslash\{x\}$ is not winning in $\Gamma\left(G^{\prime}\right)$. When $X=V\left(G^{\prime}\right), X \backslash\{x\}$ is winning in $\Gamma\left(G^{\prime}\right)$ and thus $X \notin C_{x}$. As a consequence, we have that $\eta_{x}(\Gamma)$ coincides with the number of vertex covers of $G$ minus one. As computing the number of vertex covers of a graph is \#P-hard, we have that BvAL is \#P-hard.

According to [5] (Theorem 3.29, page 50), to prove that SSvaL is \#P-hard, it is enough to show that BvaL is \#Phard and that influence games satisfy the property of being a reasonable representation. This last condition is stated as follows. For a simple game $\Gamma=(N, \mathcal{W})$, consider the game $\Gamma^{\prime}=\left(N \cup\{x\}, \mathcal{W}^{\prime}\right)$, where $x$ is a new player and $\mathcal{W}^{\prime}=\{S \cup\{x\} \mid S \in \mathcal{W}\}$. A representation of simple games is reasonable if a representation of the game $\Gamma^{\prime}$ can be computed with only polynomial blow-up with respect to a given representation of the game $\Gamma$. In the remaining of this proof we show that influence games are a reasonable representation.

Let $\Gamma=(G, w, f, q, N)$ be an influence game, and assume that $G=(V, E)$ has $n$ vertices and $m$ edges. Consider the influence graph $\left(G^{\prime}, w^{\prime}, f^{\prime}\right)$ where

- $G^{\prime}=\left(V^{\prime}, E^{\prime}\right)$ and $V^{\prime}=V \cup\{x, y\} \cup\left\{a_{1}, \ldots, a_{2 n}\right\}$,

- $E^{\prime}=E \cup\{(x, y)\} \cup\{(v, y) \mid v \in V\} \cup\left\{\left(y, a_{i}\right) \mid 1 \leq i \leq 2 n\right\}$,

- $w^{\prime}(e)=w(e)$, for any $e \in E$, and $w^{\prime}(e)=1$, for any $e \in E^{\prime} \backslash E$, and

- $f^{\prime}(v)=f(v)$, for any $v \in V, f^{\prime}(x)=1, f^{\prime}(y)=q+1$, and $f^{\prime}\left(a_{i}\right)=1$, for any $1 \leq i \leq 2 n$.

Finally, we consider the influence game $\Gamma^{+}=\left(G^{\prime}, w^{\prime}, f^{\prime}, q^{\prime}, N^{\prime}\right)$ where $q^{\prime}=2 n$ and $N^{\prime}=N \cup\{x\}$.

From the previous construction, it follows that all the winning coalitions in $\Gamma^{+}$must include $x$. Furthermore, $X \cup\{x\}$ is a winning coalition in $\Gamma^{+}$if and only if $X$ is a winning coalition in $\Gamma$. Therefore, $\Gamma^{+}$is a representation of $\Gamma^{\prime}$ and has polynomial size with respect to the size of $\Gamma$. So, we conclude that influence games are a reasonable representation.

Now we consider another set of problems that reflect fundamental properties of simple games. The input to the following problems is a game $\Gamma$.

IsProper: $\quad$ Determine whether $\Gamma$ is proper, i.e., whether for every $X \in \mathcal{W}$ it holds that $N \backslash X \in \mathcal{L}$.

IsStrong: $\quad$ Determine whether $\Gamma$ is strong, i.e., whether for every $X \in \mathcal{L}$ it holds that $N \backslash X \in \mathcal{W}$.

IsDecisive: $\quad$ Determine whether $\Gamma$ is decisive, i.e., whether $X \in \mathcal{W}$ if and only if $N \backslash X \in \mathcal{L}$. 
When a simple game $\Gamma$ is given by $\left(N, \mathcal{W}^{m}\right)$, it is known that the IsProper problem can be decided in polynomial time. It suffices to check whether for every $X \in \mathcal{W}^{m}$ it holds that $N \backslash X \notin \mathcal{W}$ in polynomial time. Further, the IsStrong problem is coNP-complete [46] and the IsDecisive problem can be solved in quasi-polynomial time [23] but is not known to be polynomial time solvable. All these results were obtained in the context of simple game theory [50, 27].

The following problems consider properties of a player with respect to an influence game. Their input is composed of a simple game $\Gamma$ and player $i$.

IsDummy: $\quad$ Determine whether player $i$ is a dummy player, i.e., whether for every team $X \in \mathcal{W}$ with $i \in X$ it holds that $X \backslash\{i\} \in \mathcal{W}$.

IsPASSER: Determine whether player $i$ is a passer, i.e., whether for every team $X$ with $i \in X$ it holds that $X \in \mathcal{W}$.

IsVetoen: $\quad$ Determine whether player $i$ is a veto player, i.e., whether for every team $X$ with $i \notin X$ it holds that $X \in \mathcal{L}$.

IsDictator: $\quad$ Determine whether player $i$ is a dictator, i.e., whether $X \in \mathcal{W}$ if and only if $i \in X$.

IsCritical: $\quad$ Given, in addition, a team $X$ including $i$, determine whether $i$ is critical for $X$, i.e., $X \in \mathcal{W}$ and $X \backslash\{i\} \in \mathcal{L}$.

AreSymmetric: $\quad$ Given, in addition, an agent $j$, determine whether players $i$ and $j$ are symmetric, i.e., whether for every team $X$ with $i, j \notin X$ it holds that $X \cup\{i\} \in \mathcal{W}$ if and only if $X \cup\{j\} \in \mathcal{W}$.

The following problems consider properties of a team of agents $X$ with respect to an influence game. Now the input is composed of a game $\Gamma$ and a team $X$.

IsBlockInG: $\quad$ Determine whether $X$ is a blocking team, i.e., whether $N \backslash X \in \mathcal{L}$.

IsSwING: $\quad$ Determine whether $X$ is a swing, i.e., whether $X \in \mathcal{W}$ but there is $i \in X$ such that $X \backslash\{i\} \in \mathcal{L}$.

The following result states those properties that can be decided in polynomial time.

Theorem 10. For influence games, the problems IsPasser, IsVetoer, IsDictator, IsCritical, IsBlocking and IsSwing belong to $\mathrm{P}$.

Proof. We provide characterizations of the properties in terms of the membership of relevant sets in either the set of winning or losing coalitions. Given an influence game $\Gamma=(G, w, f, q, N), i \in N$ and $X \subseteq N$, we have.

- Player $i$ is a passer if and only if $\{i\}$ is winning in $\Gamma$.

- Player $i$ is a vetoer if and only if $N \backslash\{i\}$ is losing in $\Gamma$.

- Player $i$ is a dictator if and only if $N \backslash\{i\}$ is losing and $\{i\}$ is winning in $\Gamma$.

- Player $i$ is critical for team $X$ if and only if $X$ is winning and $X \backslash\{i\}$ is losing in $\Gamma$.

- Team $X$ is blocking if and only if $N \backslash X$ is losing in $\Gamma$.

- Team $X$ is a swing if and only if $X$ is winning in $\Gamma$ and there is $i \in X$ for which $X \backslash\{i\}$ is $\operatorname{losing}$ in $\Gamma$.

From Theorem 6 we know that deciding if a coalition is winning can be decided in polynomial time. Therefore, we get the claimed result. 


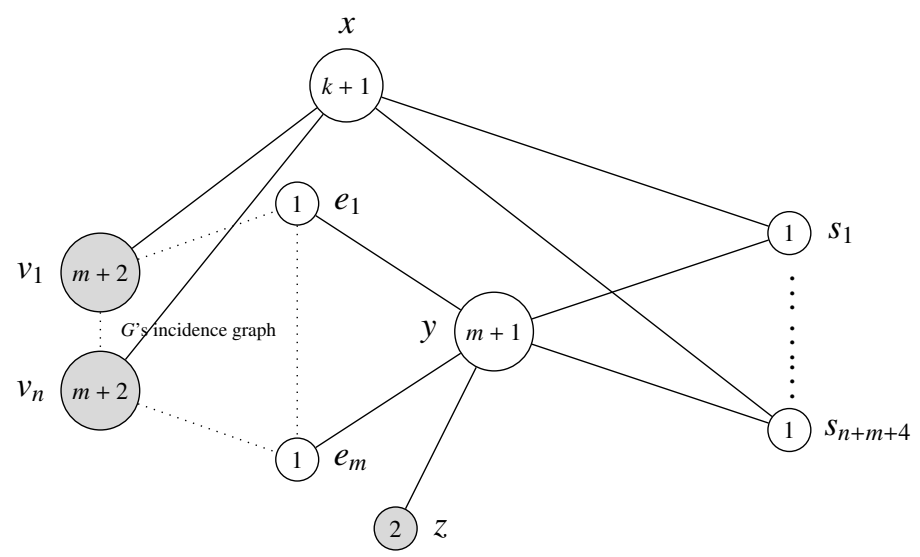

Figure 8: Influence graph $\left(G_{1}, f_{1}\right)$ of the game $\Delta_{1}(G, k)$.

To analyze the complexity of the remaining problems, we consider a new construction. Let $G=(V, E)$ be a graph where $V=\left\{v_{1}, \ldots, v_{n}\right\}$ and $E=\left\{e_{1}, \ldots, e_{m}\right\}$, and let $k$ be an integer (which will be the target size of the set cover). Then the unweighted influence game $\Delta_{1}(G, k)=\left(G_{1}, f_{1}, q_{1}, N_{1}\right)$ is defined as follows, where Figure 8 shows the corresponding influence graph.

The graph $G_{1}=\left(V_{1}, E_{1}\right)$ has $V_{1}=\left\{v_{1}, \ldots, v_{n}, e_{1}, \ldots, e_{m}, x, y, z, s_{1}, \ldots, s_{\alpha}\right\}$ where $\alpha=m+n+4$. The edges in $E_{1}$ are constructed as follows. We incorporate the incidence graph of $G$ : for any $e=\left(v_{i}, v_{j}\right) \in E$, we add to $E_{1}$ the edges $\left(e, v_{i}\right),\left(e, v_{j}\right)$ and $(e, y)$. For any $1 \leq i \leq n$, we add the edge $\left(v_{i}, x\right)$. For any $1 \leq j \leq \alpha$, we add the edges $\left(x, s_{j}\right)$ and $\left(y, s_{j}\right)$. Finally, we add the edge $(z, y)$. The labeling function $f_{1}$ is defined as: $f_{1}\left(v_{i}\right)=m+2,1 \leq i \leq n ; f_{1}\left(e_{j}\right)=1$, $1 \leq j \leq m ; f_{1}\left(s_{\ell}\right)=1,1 \leq \ell \leq \alpha$; and $f_{1}(z)=2, f_{1}(x)=k+1, f_{1}(y)=m+1$. The quota is $q_{1}=\alpha$ and the set of players is $N_{1}=\left\{v_{1}, \ldots, v_{n}, z\right\}$.

Observe that by construction the game $\Delta_{1}(G, k)$ can be obtained in polynomial time. As an immediate consequence of the definition, we have that $X$ is a successful team in $\Delta_{1}(G, k)$ if and only if either $(|X \cap V| \geq k+1)$ or $z \in X$ and $X \backslash z$ is a vertex cover in $G$.

Our next result settles the complexity of the problems which are coNP-complete.

Theorem 11. For unweighted influence games with polynomial number of vertices, the problems AReSYMmetric, IsDummy, IsProper, IsStrong and IsDecisive are coNP-complete.

Proof. Membership in coNP follows from the definitions. To get the hardness results, we provide reductions from the complement of the Vertex Cover problem and some other problems derived from it. Let $(G, k)$ be an input to VerTeX Cover, as usual we assume that $G$ has $n$ vertices and $m$ edges.

Let us start by considering the IsDummy problem. Starting from $G=(V, E)$ and $k$, we construct the unweighted influence game $\Delta_{1}(G, k)$ and the pair $\left(\Delta_{1}(G, k), z\right)$ which is an instance of the IsDummy problem. If $G$ has a vertex cover $X$ with size $k$ or less, by construction, we have that $X \cup\{z\}$ is a successful team in $\Delta_{1}(G, k)$. Furthermore, if $X$ is a vertex cover of minimum size, we have that $X \cup\{z\}$ is a minimal successful team. Therefore, $z$ is not a dummy player in $\Delta_{1}(G, k)$. If $G$ does not have a vertex cover with size $k$ or less and $X$ is a successful team containing $z$, it must hold that $|X \backslash\{z\}|>k$, therefore $X \backslash\{z\}$ is a successful team. In consequence, $z$ is a dummy player in $\Delta_{1}(G, k)$. As the pair $\left(\Delta_{1}(G, k), z\right)$ is computable in polynomial time, we have the desired result.

Let us now consider the ARESYmmetric problem. Starting from $G=(V, E)$ and $k$, we construct the unweighted influence game $\Delta_{2}(G, k)=\left(G_{2}, f_{2}, q_{2}, N_{2}\right)$ (see Figure 9). $G_{2}$ is obtained from the graph $G_{1}$ appearing in the construction of $\Delta_{1}(G, k)$ by adding two new vertices $t$ and $s$ and the edges $(x, s),(y, s)$ and $(t, s)$. Recall that $V\left(G_{1}\right)=$ $\left\{v_{1}, \ldots, v_{n}, e_{1}, \ldots, e_{m}, x, y, z, s_{1}, \ldots, s_{\alpha}\right\}$. The label function is the following: $f_{2}(v)=f_{1}(v)$, for $v \in V\left(G_{2}\right) \cap V\left(G_{1}\right)$; $f_{2}(s)=4 ; f_{2}(t)=2$. Finally, $q_{2}=\alpha+1=n+m+5$ and $N_{2}=\left\{v_{1}, \ldots, v_{n}, z, t\right\}$. Note that a description of $G_{2}$ can be obtained in polynomial time as well as a description of $\Delta_{2}(G, k)$ given a description of $(G, k)$. Let us show that the construction is indeed a reduction. Consider the tuple $\left(\Delta_{2}(G, k), z, t\right)$ which is an instance of the AreSymmetric problem. 


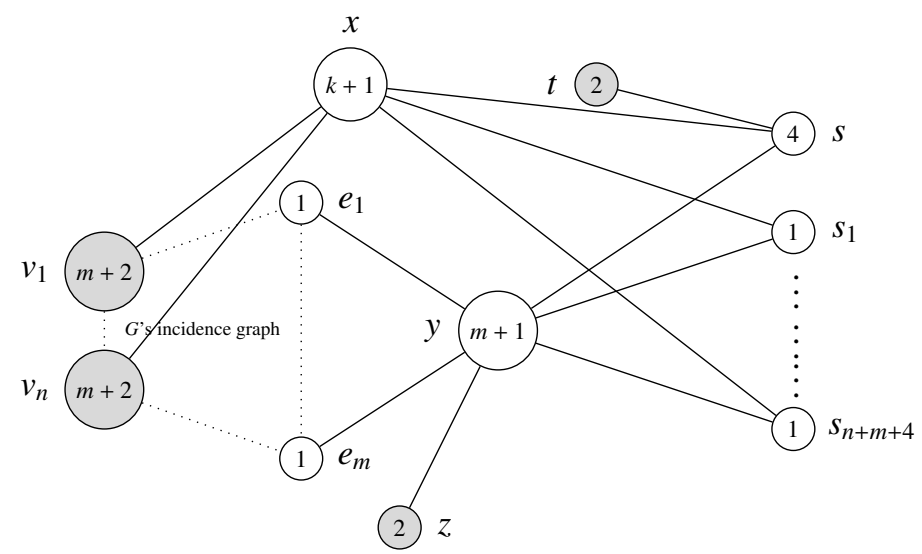

Figure 9: Influence graph $\left(G_{2}, f_{2}\right)$ used in the definition of the game $\Delta_{2}(G, k)$.

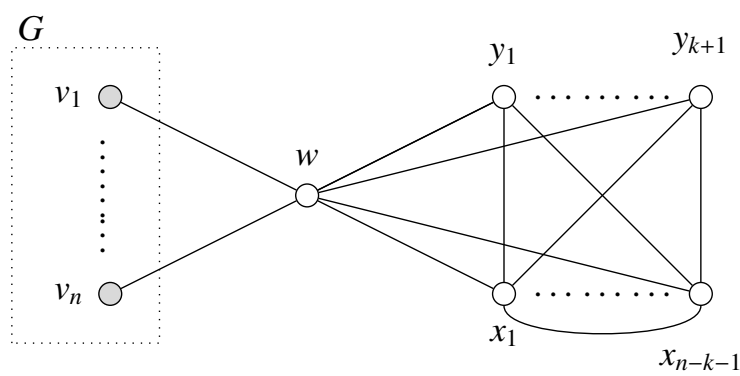

Figure 10: Graph $\hat{G}$ used to prove that HALF VERTEX COVER is NP-hard.

When $G$ has a vertex cover $X$ of size $k$ or less, by construction the team $X \cup\{z\}$ is successful in $\Delta_{2}(G, k)$ while the team $X \cup\{t\}$ is unsuccessful. Therefore $z$ and $t$ are not symmetric. When $G$ does not have a vertex cover $X$ of size $k$ or less, by construction, any successful team $Y$ must contain a subset with at least $k+1$ vertices from $\left\{v_{1}, \ldots, v_{n}\right\}$. Therefore both $Y \cup\{z\}$ and $Y \cup\{t\}$ are successful teams in $\Delta_{2}(G, k)$, i.e., vertices $z$ and $t$ are symmetric.

To prove hardness of the next two problems, IsProper and IsDecisive, we provide a reduction from the following variation of the VerTEx COVER problem:

HALF Vertex cover: Given an undirected graph with an odd number of vertices $n$. Is there a vertex cover with size $(n-1) / 2$ or less?

We first show that the HALF VERTEX COVER problem is NP-complete. By definition the problem belongs to NP. To prove hardness we show a reduction from the Vertex Cover problem. Given a graph $G$ with $n$ vertices and an integer $k$, $0 \leq k \leq n$, we construct a graph $\hat{G}$ as follows (see Figure 10). $\hat{G}$ has vertex set $\hat{V}=V(G) \cup X \cup Y \cup\{w\}$, where $X$ has $n-k-1$ vertices, $Y$ has $k+1$ vertices, and edge set

$$
\begin{aligned}
\hat{E}=E & \cup\left\{\left(x, x^{\prime}\right) \mid x \neq x^{\prime} \wedge x, x^{\prime} \in X\right\} \\
& \cup\{(x, y) \mid x \in X, y \in Y\} \\
& \cup\{(w, z) \mid z \in V \cup X \cup Y\}
\end{aligned}
$$

By construction, $\hat{G}$ has $2 n+1$ vertices, so it can be constructed in polynomial time. Note that any vertex cover $S$ of $\hat{G}$ with minimum size has to contain $w$, all the vertices in $X$ and no vertex from $Y$. The rest of the cover, $S \cap V$ must 


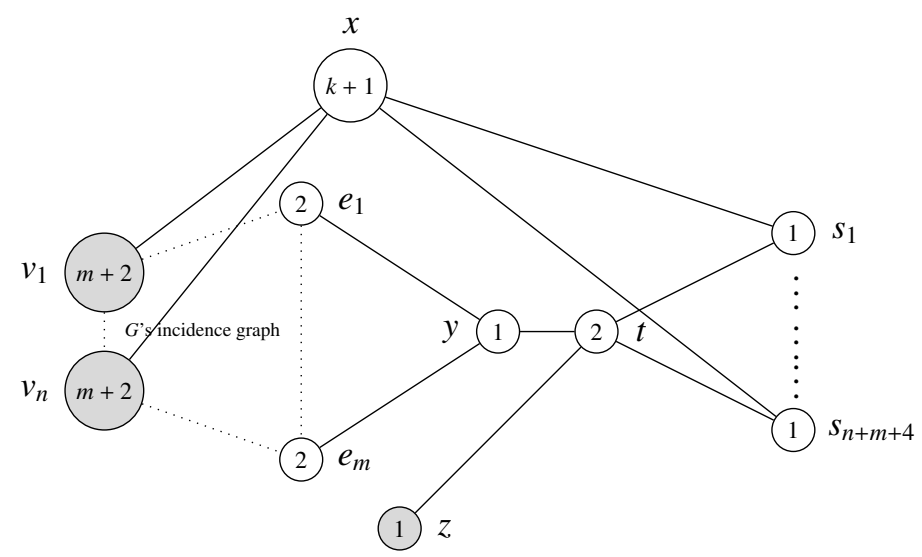

Figure 11: Influence graph $\left(G_{3}, f_{3}\right)$ of the game $\Delta_{3}(G)$.

be a minimum vertex cover of $G$. Therefore, $G$ has a vertex cover of size $k$ or less if and only if $\hat{G}$ has a vertex cover of size $n$ or less.

Let us provide a reduction from the complement of HALF VERTEX COVER to the ISPROPER and the IsDecisive problems. Let $G$ be an instance of HALF VERTEX COVER with $n=2 k+1$ vertices, for some value $k \geq 1$. Consider the unweighted influence game $\Delta_{1}(G,(n-1) / 2)=\left(G_{1}, f_{1}, q_{1}, N_{1}\right)$. Recall that $V\left(G^{\prime}\right)=\left\{v_{1}, \ldots, v_{n}, e_{1}, \ldots, e_{m}, x, y, z, s_{1}, \ldots, s_{\alpha}\right\}$ where $q_{1}=\alpha=n+m+4$ and $N_{1}=\left\{v_{1}, \ldots, v_{n}, z\right\}$.

If $G$ has a vertex cover $X$ with $|X| \leq k$, the team $X \cup\{z\}$ is successful and, as $n+1-|X \cup\{z\}|>k$, we have that $N \backslash(X \cup\{z\})$ is also successful. Hence $\Delta_{1}(G, k)$ is not proper. When all the vertex covers of $G$ have more than $k$ vertices, any successful team $Y$ of $\Delta_{1}(G, k)$ satisfies $\left|Y \cap\left\{v_{1}, \ldots, v_{n}\right\}\right|>k$, i.e., $\left|Y \cap\left\{v_{1}, \ldots, v_{n}\right\}\right| \geq k+1$. For a successful team $Y$, we have to consider two cases: $z \in Y$ and $z \notin Y$. When $z \in Y, N \backslash Y \subseteq\left\{v_{1}, \ldots, v_{n}\right\}$ and $|N \backslash Y| \leq n-k-1=k$. Thus, $N \backslash Y$ is an unsuccessful team. When $z \notin Y,|N \backslash(Y \cup\{z\})| \leq k$ and $N \backslash Y$ is again an unsuccessful team. So, we conclude that $\Delta_{1}(G,(n-1) / 2)$ is proper. As $\Delta_{1}(G,(n-1) / 2)$ can be obtained in polynomial time, the IsProper problem is coNP-hard.

Observe that when $G$ is an instance of the HALF VERTEX COVER and all the vertex covers of $G$ have more than $(n-1) / 2$ vertices, the game $\Delta_{1}(G,(n-1) / 2)$ is also decisive. When this condition is not met, the game $\Delta_{1}(G,(n-1) / 2)$ is not proper and thus it is not decisive. Thus, we conclude that the IsDecisive problem is also coNP-hard.

To finish the proof we show hardness for the IsSTRONG problem. We provide a reduction from the complement of the following problem.

HALF INDEPENDENT SET: Given an undirected graph with an even number of vertices $n$.

Is there an independent set with size $n / 2$ or higher?

The HaLf INDEPENDENT SET trivially belongs to NP. Hardness follows from a simple reduction from Vertex Cover. Starting from a graph $G$ with an odd number of vertices we construct a new graph $G^{\prime}$ by adding one new vertex connected to all the vertices in $G$. This construction guarantees that $G$ has a vertex cover of size $(n-1) / 2$ or less if and only if $G^{\prime}$ has a vertex cover with size $n / 2$ or less. As the complement of a vertex cover is an independent set, we have that $G$ has a vertex cover of size $(n-1) / 2$ or less if and only if $G^{\prime}$ has an independent set with size $n / 2$ or higher.

Now we show that the complement of the HALF INDEPENDENT SET problem can be reduced to the IsSTRONG problem. We associate to an input to HALF INDEPENDENT SET the game $\Delta_{3}(G)=\left(G_{3}, f_{3}, n+m+5, N_{3}\right)$ where $N_{3}=V \cup\{z\}$ and $\left(G_{3}, f_{3}\right)$ is the influence graph described in Figure 11. This game is a variation of $\Delta_{1}(G, k)$ using ideas similar to those in the reduction from the Set PAcKing problem in Theorem 8.

When $G$ has an independent set with size at least $n / 2, G$ also has an independent set $X$ with $|X|=n / 2$. It is easy to see that both the team $X \cup\{z\}$ and its complement are unsuccessful in $\Delta_{3}(G)$. Therefore, $\Delta_{3}(G)$ is not strong. Assume now that all the independent sets in $G$ have less than $n / 2$ vertices. Observe that, for a team $X$ in $\Delta_{3}(G)$ with $|X \cap V|<n / 2$, its complement has at least $n / 2+1$ elements in $V$ and thus it is successful. When $|X \cap V|>n / 2$ the team is successful. Therefore we have to consider only those teams with $|X \cap V|=n / 2$. In such a case, we know that 
neither $X \cap V$ nor $V \backslash(X \cap V)$ are independent sets. Then, by construction, one of the sets $X$ or $N \backslash X$ must contain $z$ and is successful while its complement is unsuccessful. In consequence $\Delta_{3}(G)$ is strong.

Now we consider the complexity of problems related to game isomorphism and equivalence. We state here the definitions for influence games.

Definition 7. Let $\Gamma=(G, w, f, q, N)$ and $\Gamma^{\prime}=\left(G^{\prime}, w^{\prime}, f^{\prime}, q^{\prime}, N^{\prime}\right)$ be two influence games with the same number of players. $\Gamma$ and $\Gamma^{\prime}$ are isomorphic if and only if there exists a bijective function, $\varphi: N \rightarrow N^{\prime}$, such that $|F(X)| \geq$ $q$ if and only if $|F(\varphi(X))| \geq q^{\prime}$. Moreover, when $N=N^{\prime}$ and $\varphi$ is the identity function, then we say that the two influence games are equivalent.

The associated problems have as input two influence games and are stated as follows.

Iso: $\quad$ Determine whether the two influence games are isomorphic.

EQuiv: $\quad$ Determine whether the two influence games are equivalent.

Theorem 12. For unweighted influence games with polynomial number of vertices, the problem EQUIV is coNPcomplete and the problem Iso is coNP-hard and belongs to $\Sigma_{2}^{p}$.

Proof. Membership to the corresponding complexity classes follows directly from the definition of the problems. For the hardness part we provide a reduction from the complement of the VerTex Cover problem. Let $G$ be a graph and consider the influence game $\Gamma_{1}=\Delta_{1}(G, k)$ as defined before (see Figure 8). Recall that the set of players is $N_{1}=\left\{v_{1}, \ldots, v_{n}, z\right\}$. To define the second influence game $\Gamma_{2}$ we consider the weighted game with set of players $N_{1}$ and quota $q=k+1$. The weights of the players are the following: $w\left(v_{i}\right)=1$, for any $1 \leq i \leq n$, and $w(z)=0$. A representation of $\Gamma_{2}$ as an unweighted influence game can be obtained in polynomial time using the construction of Theorem 3. Our reduction associates to an input to vertex cover $(G, k)$ the pair of influence games $\left(\Gamma_{1}, \Gamma_{2}\right)$. Observe that $\Gamma_{1}$ is equivalent (isomorphic) to $\Gamma_{2}$ if and only if $G$ does not have a vertex cover of size $k$ or less.

We have been unable to provide a complete classification for the Iso problem. It remains open to show whether this problem is $\Sigma_{2}^{p}$-hard or not.

\section{Unweighted Influence Games on Undirected Graphs}

In this section we analyze the complexity of the proposed problems on some particular subfamilies of unweighted influence games defined on undirected graphs.

\subsection{Maximum Influence}

Here we analyze first the case with maximum influence and maximum spread, that is games of the form $\Gamma=$ $(G, f,|V|, V)$ where $f(v)=d_{G}(v)$, or, as we said before, the game $\Gamma=\Gamma(G)$, for some graph $G$. When the graph $G$ is disconnected with connected components $C_{1}, \ldots, C_{k}$, the associated game $\Gamma(G)$ can be analyzed from the $\Gamma\left(C_{1}\right), \ldots, \Gamma\left(C_{k}\right)$. Observe that, due to maximum spread, a successful team must influence all the vertices in the graph. Therefore, the members of a successful team in a connected component must influence all the vertices in their component. So, a team $X$ is successful in $\Gamma(G)$ if and only if, for any $1 \leq i \leq k$, the team $X \cap V\left(C_{i}\right)$ is successful in $\Gamma\left(C_{i}\right)$. We analyze first the case in which $G$ is connected.

Lemma 3. In an unweighted influence game $\Gamma$ with maximum influence and maximum spread on a connected graph $G$ the following properties hold.

- $\Gamma$ is proper if and only if $G$ is either not bipartite or a singleton.

- $\Gamma$ is strong if and only if $G$ is either a star or a triangle.

- $\Gamma$ is decisive if and only if $G$ is a triangle. 
Proof. From Lemma 2 we know that the successful teams of $\Gamma=\Gamma(G)$ coincide with the vertex covers of $G$. We also recall that the complement of a vertex cover is an independent set.

If $G=(V, E)$ is bipartite, let $\left(V_{1}, V_{2}\right)$ be a partition of $V$ so that $V_{1}$ and $V_{2}$ are independent sets. In such a case, both $V_{1}$ and $V_{2}=N \backslash V_{1}$ are successful teams in $\Gamma$. Therefore, $\Gamma$ is not proper. For the opposite direction, if $\Gamma$ is not proper, then the game admits two disjoint successful teams, i.e, two disjoint vertex covers of $G$, and hence each of them must be an independent set. Thus the graph $G$ is bipartite.

Now we prove that $\Gamma$ is not strong if and only if $G$ has at least two non-incident edges. Observe that a graph where all edges are incident is either a triangle or a star. If $G$ has at least two non-incident edges $e_{1}=\left(u_{1}, v_{1}\right)$ and $e_{2}=\left(u_{2}, v_{2}\right),\left\{u_{1}, v_{1}\right\}$ and $N \backslash\left\{u_{1}, v_{1}\right\}$ are both unsuccessful teams, therefore $\Gamma$ is not strong. When the game is not strong, there is a team $X$ such that both $X$ and $N \backslash X$ are unsuccessful. For this to happen it must be that there is an edge uncovered by $X$ and another edge uncovered by $N \backslash X$. Thus $G$ must have two non-incident edges.

Finally, it is well known that a non-bipartite graphs has at least one odd cycle, so the only non-bipartite graph with all pair of edges incidents (proper and strong) is a triangle (decisive).

In this kind of influence games, when the graph of a game $\Gamma$ is disconnected, a team $X$ is successful in $\Gamma$ if and only if, for every $1 \leq i \leq k$, the team $X \cap V\left(C_{i}\right)$ is successful in $\Gamma\left(C_{i}\right)$. By using this consideration, the previous result can be extended to disconnected graphs. We can also provide characterizations for other properties for graphs connected or not.

Lemma 4. In an unweighted influence game $\Gamma$ with maximum influence and maximum spread on a graph $G$ the following properties hold.

- $\Gamma$ is proper if and only if at least one connected component of $G$ is either not bipartite or a singleton.

- $\Gamma$ is neither strong nor decisive if $G$ has at least two connected components.

- Г has no dummy players.

- Two players $i$ and $j$ are symmetric in $\Gamma$ if and only if $N(i) \backslash\{j\}=N(j) \backslash\{i\}$.

Proof. For the first property, let $C_{1}$ be a connected component of $G$. Suppose that at least $C_{1}$ is not bipartite, and consider a team $X$ which is successful in $\Gamma$. Since the team $X \cap V\left(C_{1}\right)$ is successful in $\Gamma\left(C_{1}\right)$, by Lemma 3 the team $V\left(C_{1}\right) \backslash X$ is unsuccessful in $\Gamma\left(C_{1}\right)$ and hence $V \backslash X$ is unsuccessful in $\Gamma$, so the game is proper. If $C_{1}$ is a singleton, then every successful team has to contain $C_{1}$, and therefore its complement cannot influence $C_{1}$ so it is unsuccessful, i.e., the game is proper.

For the second property, suppose that there are at least two connected components of $G$, namely $C_{1}$ and $C_{2}$. Since the team $V \backslash C_{1}$ cannot influence $C_{1}$, then it is unsuccessful, but its complement $C_{1}$ is unsuccessful as well, because it cannot influence $C_{2}$. Therefore, the game is neither strong nor decisive.

For the third property, let $G=(V, E)$ be a graph and let $i \in V$ be a player in $\Gamma(G)$. When $i$ is an isolated vertex, $V$ is winning but $V \backslash\{i\}$ is losing. Otherwise, there must be a vertex $j$ with $(i, j) \in E$. In this case $V \backslash\{j\}$ is winning but $V \backslash\{i, j\}$ is losing. Therefore $i$ is not dummy.

Finally, to prove the last property we consider four cases.

Case 1: $N(i) \backslash\{j\} \neq N(j) \backslash\{i\}$ and $N(i) \backslash\{j\}=\emptyset$ or $N(j) \backslash\{i\}=\emptyset$. In this case we can assume that $N(j) \backslash\{i\}=\emptyset$ and $N(i) \backslash\{j\} \neq \emptyset$ without loss of generality. Let $k \in N(i), k \neq j$. If $i \in N(j)$ then $N(j)=\{i\}$, so taking $X=V \backslash\{i, j, k\}$ we have that $X \cup\{i\}$ is winning while $X \cup\{j\}$ is losing. If $i \notin N(j)$ then $N(j)=\emptyset$, so taking $X=V \backslash\{i, j\}$ it is clear that $X \cup\{j\}$ is winning, but $X \cup\{i\}$ is losing. Therefore $i$ and $j$ are not symmetric.

Case 2: $N(i) \backslash\{j\} \neq N(j) \backslash\{i\}, N(i) \backslash\{j\} \neq \emptyset$ and $N(j) \backslash\{i\} \neq \emptyset$. Without loss of generality, we can assume that there exists a vertex $k$ with $(i, k) \in E$ and $(j, k) \notin E$. Let $X=V \backslash\{i, j, k\}$. Observe that as both $N(j)$ and $N(k)$ are subsets of $X \cup\{i\}, X \cup\{i\}$ is winning. But as neither $i$ nor $k$ belong to $X \cup\{j\}, X \cup\{j\}$ is losing. Therefore $i$ and $j$ are not symmetric.

Case 3: $N(i) \backslash\{j\}=N(j) \backslash\{i\}=\emptyset$. Let $X$ be a subset of $V \backslash\{i, j\}$. If $(i, j) \notin E$, both $X \cup\{i\}$ and $X \cup\{j\}$ are losing. If $(i, j) \in E$, both $X \cup\{i\}$ and $X \cup\{j\}$ are winning. Therefore, $i$ and $j$ are symmetric. 
Case 4: $N(i) \backslash\{j\}=N(j) \backslash\{i\} \neq \emptyset$. Let $X$ be a subset of $V \backslash\{i, j\}$. As the winning coalitions correspond to the vertex covers, it follows that $X \cup\{i\}$ is a winning coalition if and only if $N(j) \backslash\{i\} \subseteq X$ and $X$ is a vertex cover of $G[V \backslash\{i, j\}]$. The condition is the same for $j$, therefore $i$ and $j$ are symmetric.

The next results follow directly from the previous lemma.

Theorem 13. For unweighted influence games with maximum influence and maximum spread, the problems IsDummY, AreSymmetric, IsProper, IsStrong, IsDecisive belong to $\mathrm{P}$.

In regard to the complexity of the two main game measures we have the following result.

Theorem 14. For unweighted influence games with maximum influence and maximum spread on a connected graph $G$, computing LENGTH is NP-hard. Computing WIDTH of $\Gamma(G)$ can be done in polynomial time even when $G$ is disconnected.

Proof. As before we use the fact that $\Gamma(G)$ can be computed in polynomial time. Furthermore, from Lemma 2 , $\operatorname{Length}(\Gamma(G))$ is the minimum size of a vertex cover of $G$. Therefore LenGth is NP-hard.

We prove that WIDTH can be computed in polynomial time by a case analysis. If $G$ is just an isolated vertex or just one edge, the empty set is the unique unsuccessful team, thus $\operatorname{WIDTH}(\Gamma)=0$. Otherwise, either $G$ has no edges or has at least one edge and an additional vertex. In the first case, the graph is an independent set with at least two vertices. Assume that $u \in V$, then $V \backslash\{u\}$ is unsuccessful and we conclude that $\mathrm{W} \operatorname{IDTH}(\Gamma)=n-1$.

In the second case $G$ has at least one edge $e=(u, v)$ and $V \backslash\{u, v\}$ is non-empty. We have again two cases, either $G$ has an isolated vertex $z$ or all the connected components of $G$ have at least one edge. When $z$ is an isolated vertex the team $V \backslash\{z\}$ is unsuccessful, therefore $\operatorname{WIDTH}(\Gamma)=n-1$. When all the connected components of $G$ have at least one edge, any team with $n-1$ nodes is a vertex cover, thus $\operatorname{WIDTH}(\Gamma)<n-1$. Observe that the set $V \backslash\{u, v\}$ is not empty and, furthermore it does not cover the edge $e$, thus we have an unsuccessful team with $n-2$ vertices. Thus, in this case $\operatorname{WIDTH}(\Gamma)=n-2$.

As the classification can be checked trivially in polynomial time we get the claimed result.

For the case of maximum influence but not maximum spread, that is influence games of the form $(G, f, q, V)$ where $f(v)=d_{G}(v)$ and $q<n$, the game cannot be directly analyzed from the games on the connected components, as the total quota can be fulfilled in different ways by the agents influenced in each component. Nevertheless, as the influence is maximum, any set of vertices $X$ can influence another vertex $u$ only when all the neighbors of $u$ are included in $X$, alternatively when $u$ becomes an isolated vertex after removing $X$. This leads to the following characterization of the successful teams.

Lemma 5. In an unweighted influence game with maximum influence $\Gamma=\left(G, d_{G}, q, V\right)$ where $G$ has no isolated vertices, $X \subseteq V$ is a successful team if and only if removing $X$ from $G$ leaves at least $q-|X|$ isolated vertices.

By considering this characterization, we have the following results.

Theorem 15. For unweighted influence games $\Gamma$ with maximum influence, LENGTH is NP-hard and WIDTH belongs to P.

Proof. The hardness result follows from Theorem 14, because here we are considering a more general class of influence games.

When computing the WIDTH of $\Gamma=\left(G, d_{G}, q, V\right)$ we want to maximize the size of the unsuccessful teams. Therefore, we can restrict ourselves to analyzing only unsuccessful teams $X$ for which $F(X)=X$. We have that $X$ is an unsuccessful team with $F(X)=X$ if and only if $|X|<q$ and every non isolated vertex in $V \backslash X$ remains non isolated in $G[V \backslash X]$ (the subgraph induced by $N \backslash X$ ).

We consider first the case in which $G$ has no isolated vertices. We first solve the problem of deciding whether, for a given $\alpha$, it is possible to discard $\alpha$ nodes from $G$ without leaving isolated vertices. For doing so we sort the sizes of the connected components of $G$ in increasing order of size. As $G$ has no isolated vertices all the connected components have at least two vertices. Assume that $G$ has $k$ connected components $C_{1}, \ldots, C_{k}$ with sizes $2 \leq w_{1} \leq w_{2} \leq \cdots \leq w_{k}$. Now we consider two cases. 
Case 1: $w_{k}=2$, all the connected components have exactly two vertices. Therefore, if $\alpha$ is even and at most $n$, we can discard the $\alpha$ vertices in the first $\alpha / 2$ components, without leaving isolated vertices. Otherwise, the removal of any set of size $\alpha$ will leave at least one isolated vertex.

Case 2: $w_{k}>2$. We compute the value $j$ for which $\sum_{i=1}^{j} w_{i} \leq \alpha$ but $\sum_{i=1}^{j+1} w_{i}>\alpha$. Let $\beta=\sum_{i=1}^{j} w_{i}$. Let $S_{j}$ be the set of vertices in the first $j$ components. If $\beta=\alpha, S_{j}$ can be removed without leaving isolated vertices. When $\beta<\alpha$ we have two cases:

(1) $w_{j+1}>\alpha-\beta+1$. Let $C \subset C_{j+1}$ be a set with $w_{j+1}-(\alpha-\beta)$ vertices such that $G[C]$ is connected. The vertices in $S_{j}$ together with the $\alpha-\beta$ vertices of $C_{j+1} \backslash C$ can be removed without leaving any isolated vertex.

(2) $w_{j+1} \leq \alpha-\beta+1$. By construction, $\alpha<\beta+w_{j+1}$, thus $w_{j+1}=\alpha-\beta+1$. If $j+1<k$, removing the vertices in $S_{j}$ together with $\alpha-\beta-1$ vertices from the $j+1$-th component (as in case (1)) and one additional vertex from the $k$-th component leaves no isolated vertices. If $j+1=k$, the removal of any set of size $\alpha$ will leave at least one isolated vertex.

The previous characterization can be decided in polynomial time for any value of $\alpha$. By performing the test for $\alpha=q-1, q-2, \ldots, 1$ we can compute in polynomial time the maximum value of $\alpha\left(\alpha_{\text {max }}\right)$ for which $\alpha$ nodes can be discarded without leaving isolated vertices. As the WIDTH of the game is just $\alpha_{\max }$ we get the desired result for graphs without isolated vertices.

When $G$ has $n_{0}$ isolated vertices, we consider the graph $G^{\prime}=\left(V^{\prime}, E^{\prime}\right)$ obtained from $G$ by removing all the isolated vertices, and $\Gamma^{\prime}=\left(G^{\prime}, d_{G^{\prime}}, q-n_{0}, V^{\prime}\right)$. Note that for a team $X$ with $X=F(X)$ and any set $Y$ of isolated vertices we have that $F(X \cup Y)=X \cup Y$, thus $\operatorname{WIDTH}(\Gamma)=\min \left\{\mathrm{W} \operatorname{IDTH}\left(\Gamma^{\prime}\right)+n_{0}, q-1\right\}$. Therefore WIDTH can be computed in polynomial time.

\subsection{Minimum Influence}

Let $\Gamma=\left(G, 1_{V}, q, N\right)$ where $1_{V}(v)=1$ for any $v \in V$. Observe that, if $G$ is connected, the game has a trivial structure as any non-empty vertex subset of $N$ is a successful team. For the disconnected case we can analyze the game with respect to a suitable weighted game. Assume that $G$ has $k$ connected components, $C_{1}, \ldots, C_{k}$. Without loss of generality, we assume that all the connected components of $G$ have non-empty intersection with $N$. For $1 \leq i \leq k$, let $w_{i}=\left|V\left(C_{i}\right)\right|$ and $n_{i}=\left|V\left(C_{i}\right) \cap N\right|$.

Lemma 6. If a successful team $X$ is minimal then it has at most one node in each connected component. Minimal successful teams are in a many-to-one correspondence with the minimal winning coalitions of the weighted game $\left[q ; w_{1}, \ldots, w_{k}\right]$.

The problems related to players that are hard in the general case can be solved in polynomial time for minimum influence games. Those algorithms use a reduction to the KNAPSACK and the 0-1-KNaPsack problems:

KNAPSACK: $\quad$ Given a finite set $U$, for each $i \in U$, a weight $w_{i}$, a value $v_{i}$, and a positive integer $k$. Find a subset $S \subseteq U$ with $\sum_{i \in S} w_{i} \leq k$ and maximum $\sum_{i \in S} v_{i}$.

0-1-KNAPSACK: $\quad$ Given a finite set $U$, for each $i \in U$, a weight $w_{i}$, and a positive integer $k$. Is there a subset $S \subseteq U$ with $\sum_{i \in S} w_{i}=k$ ?

It is well known that both the KNAPSACK and the 0-1-KNAPSACK problems can be solved in pseudo polynomial time. Therefore, when all the weights are at most $|U|$, it can be solved in polynomial time.

Theorem 16. For unweighted influence games with minimum influence, the problems LengTh, WIDTH, IsDummy, AreSymmetric, IsProper, IsStrong and IsDecisive belong to $\mathrm{P}$.

Proof. Let $\Gamma=\left(G, 1_{V}, q, N\right)$ be an unweighted influence game with minimum influence.

First we prove that LeNGTH can be computed in polynomial time. Assume that the connected components of $G$ are sorted in such a way that $w_{1} \geq \cdots \geq w_{k}$. To minimize the size of a winning coalition we consider only those coalitions with at most one player in a connected component. Observe that the $\operatorname{LENGTH}(\Gamma)$ is the minimum $j$ for which $\sum_{i=1}^{j} w_{i} \geq q$ but $\sum_{i=1}^{j-1} w_{i}<q$. Of course this value can be computed in polynomial time. 
For computing WIDTH observe that an unsuccessful team of maximum size can be obtained by computing a selection $S \subseteq\{1, \ldots, k\}$ of connected components in such a way that $\sum_{i \in S} w_{i}<q$ and $\sum_{i \in S} n_{i}$ is maximized. Computing such selection is equivalent to solving a KNAPSACK problem on a set of $k$ items, item $i$ having weight $w_{i}$ and value $n_{i}$, and setting the knapsack capacity to $q$.

Now we consider the IsDummy problem. Let $i \in N$ and assume that $i \in C_{\ell}$. Consider a set $X \subseteq N$ with $i \in X$ and let $Y=X \backslash\{i\}$. We have to consider two cases.

Case 1: $Y \cap C_{\ell} \neq \emptyset$. In such a case $F(Y)=F(X)$, so when $X \in \mathcal{W}$ we also have $Y \in \mathcal{W}$.

Case 2: $Y \cap C_{\ell}=\emptyset$. In such a case $F(X)=F(Y) \cup F(\{i\})$, so $|F(X)|=|F(Y)|+w_{\ell}$. For $X$ to be winning and $Y$ to be losing we need a set $Y$ having $|F(Y)|+w_{\ell} \geq q$ and $|F(Y)|<q$.

Therefore player $i$ is a dummy player in $\Gamma$ if and only if there is no set $Y$ with $Y \cap C_{\ell}=\emptyset$, for which $q-w_{\ell} \leq$ $|F(Y)|<q$. This last condition can be checked in polynomial time by solving a set of $w_{\ell}$ instances of the 0-1-KNAPSACK problem with object weights $\left\{w_{i} \mid i \neq \ell\right\}$ and knapsack capacities $q-w_{\ell}, \ldots, q-1$.

Let us prove that the AreSymmetric problem can be solved in polynomial time. Let $i, j \in N$ and assume that $i \in C_{\ell(i)}$ and $j \in C_{\ell(j)}$. Consider a set $Y \subseteq N$ with $i, j \notin Y$. When $\ell(i)=\ell(j), F(Y \cup\{i\})=F(Y \cup\{j\})$ and thus $i$ and $j$ are indeed symmetric. Assume now that $\ell(i) \neq \ell(j)$. Again we have to consider some cases.

Case 1: $Y \cap C_{\ell(i)}=Y \cap C_{\ell(j)}=\emptyset$. In this case we have $|F(Y \cup\{i\})|=|F(Y)|+w_{\ell(i)}$ and $|F(Y \cup\{j\})|=|F(Y)|+w_{\ell(j)}$. Thus the two players are symmetric if and only if there is no set $Y$ with $|F(Y)|+w_{\ell(i)}<q$ and $|F(Y)|+w_{\ell(j)} \geq q$ or $|F(Y)|+w_{\ell(i)} \geq q$ and $|F(Y)|+w_{\ell(j)}<q$. If $w_{\ell(i)}=w_{\ell(j)}$, then it is clear that $i$ and $j$ are symmetric. Assuming that $w_{\ell(i)}<w_{\ell(j)}$, the only possibility is $|F(Y)|+w_{\ell(i)}<q$ and $|F(Y)|+w_{\ell(j)} \geq q$, the condition can be checked again solving a polynomial number of instances of the 0-1-KNAPSACK problem with polynomial weights.

Case 2: $Y \cap C_{\ell(i)} \neq \emptyset$ and $Y \cap C_{\ell(j)} \neq \emptyset$. Here again we have $F(Y \cup\{i\})=F(Y \cup\{j\})$ therefore $i$ and $j$ are symmetric players.

Case 3: $Y \cap C_{\ell(i)}=\emptyset$ and $Y \cap C_{\ell(j)} \neq \emptyset$. Now we have $|F(Y \cup\{i\})|=|F(Y \cup\{j\})|+w_{\ell(i)}=|F(Y)|+w_{\ell(i)}$. So, we have again to check that it is impossible to have a set $Y$ verifying the condition with $|F(Y \cup\{i\})| \geq q$ and $|F(Y \cup\{j\})|<q$. Again the condition can be checked in polynomial time solving a suitable set of 0-1-KNAPSACK instances.

The remaining case $Y \cap C_{\ell(i)} \neq \emptyset$ and $Y \cap C_{\ell(j)}=\emptyset$ is symmetric to Case 3. Therefore the claim follows.

For the IsProper problem it is enough to check whether the game is not proper, that is, whether there is a winning coalition whose complement is also winning. For doing so we separate the connected components in two sets: those containing one player and those containing more that one player. Let $A=\left\{i \mid n_{i}=1\right\}$ and $B=\left\{i \mid n_{i}>1\right\}$. Let $N_{A}=\cup_{i \in A}\left(N \cap V\left(C_{i}\right)\right)$ and $N_{B}=N \backslash N_{A}$. Let $w_{A}=\sum_{i \in A} w_{i}$ and $w_{B}=w_{N}-w_{A}$. As all the components in $B$ have at least two vertices, we can find a set $X \subseteq N_{B}$ such that $|F(X)|=\left|F\left(N_{B} \backslash X\right)\right|=w_{B}$. Thus if $w_{B} \geq q$ the game is not proper. When $w_{B}<q$ the game is proper if and only if the influence game $\Gamma^{\prime}$ played on the graph formed by the connected components belonging to $A$ and quota $q^{\prime}=q-w_{B}$ is proper. Observe that $\Gamma^{\prime}$ is equivalent to the weighted game with a player for each component in $i \in A$ with associated weight $w_{i}$ and quota $q^{\prime}$.

Let $\alpha_{\min }$ be the minimum $\alpha \in\left\{q^{\prime}, \ldots, w_{A}\right\}$ for which there is a set $S \subseteq A$ with $\sum_{i \in S} w_{i}=\alpha$. Observe that $\Gamma^{\prime}$ is proper if and only if $w_{A}-\alpha_{\min }<q^{\prime}$. The value $\alpha_{\min }$ can be computed by solving several instances of the 0-1-KNAPSACK problem having item weights polynomial in $n$. Therefore, $\alpha_{\min }$ can be computed in polynomial time and the claim follows.

Now we prove that the IsStrong problem belongs to P. Observe that in order to minimize the influence of the complement of a team $X$ it is enough to consider only those teams $X$ that contain all or none of the players in a connected component. Let $w_{N}=\sum_{i=1}^{k} w_{i}$, and let $\alpha_{\max }$ be the maximum $\alpha \in\{0, \ldots, q-1\}$ for which there is a set $S \subseteq\{1, \ldots, k\}$ with $\sum_{i \in S} w_{i}=\alpha$. Note that $\alpha$ can be zero and thus $S$ can be the empty set. Observe that $\Gamma$ is strong if and only if $w_{N}-\alpha_{\max } \geq q$. The value $\alpha_{\max }$ can be computed by solving several instances of the 0 - 1 -KNAPSACK problem. As the weights are at most $n$, the value can be obtained in polynomial time.

Finally, the result for IsDecisive follows from those for IsProper and IsStrong.

\section{Conclusions}

In this paper we have considered an influence spread model in social networks as a mechanism for emergence of cooperation. Our model is based on the linear threshold model for influence spread. In such a context we use 


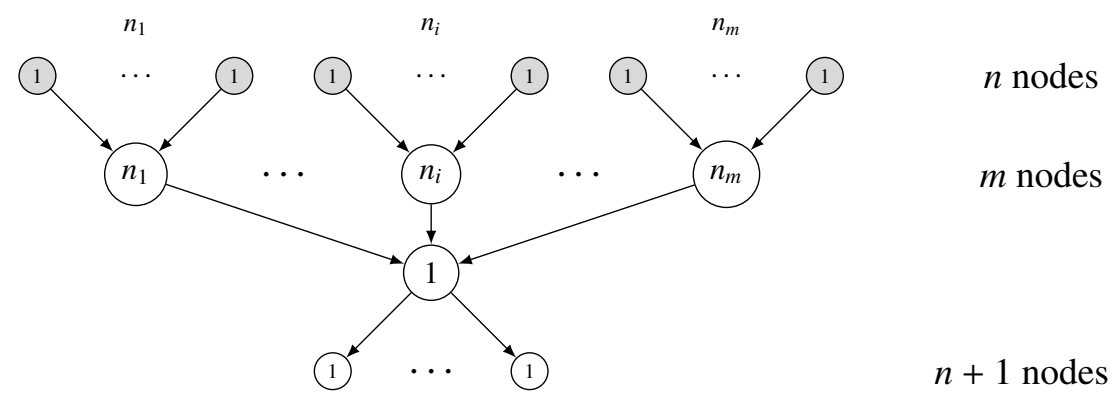

Figure 12: The simple game whose set of players $N=\{1, \ldots, n\}$ admits a partition $N_{1}, \ldots, N_{m}$ in such a way that $\mathcal{W}=\left\{S \subseteq N\right.$; $\exists N_{i}$ with $\left.N_{i} \subseteq S\right\}$ has exponential dimension, $n_{1} \cdot \ldots \cdot n_{m-1}$ [28], but this game admits a polynomial unweighted influence graph $(G, f)$ with respect to $n$ for the corresponding unweighted influence game $(G, f, n+1, N)$.

parameters and properties derived from voting systems (simple games) to analyze the properties of the system. This point of view has given rise to the introduction of a new family of simple games, called influence games. We have shown that influence games are sufficiently expressive to represent the complete family of simple games. Interestingly enough we have shown that representations, as influence games, of the union or intersection of two influence games can be computed in polynomial time. We have also shown that influence games in which the number of agents is polynomial in the number of players are a proper subset of simple games.

The remaining contribution of this paper concerns the computational complexity of problems related to several parameters and properties. Our results are summarized in Table 1. As a side result we show that computing the width of a game given by the set of minimal winning coalitions is coNP-hard, which was posted as an open problem in [5]. Finally, we have analyzed two extreme cases for the required level of influence, maximum and minimum, showing that the computational complexity of some of the considered problems changes, becoming in general more tractable. In particular we have shown a graph characterization of the proper and strong properties in terms of graph properties for influence graphs with maximum influence and maximum spread. It remains open such characterization for influence graphs with just maximum influence.

We have studied the isomorphism and equivalence problems for influence games. It remains open to show whether the Iso problem is $\Sigma_{2}^{p}$-complete. The equivalence problem has been studied for weighted and multiple weighted game [20] (see Table 1). To the best of our knowledge, the complexity of the Iso problem remains open for weighted games as well as for any of the families of simple games defined by boolean combinations of weighted games considered in this paper.

There are many open lines for future research, here we mention a few. On the general topic of spreading influence, it will be interesting to analyze the properties of the influence games defined through other influence spread mechanisms, in particular for randomized models like the independent cascade model [39]. Besides the extreme situations previously mentioned, there are many other natural rules to study, such as the majority rule, when individuals are convinced when a majority of their neighbors are. It will be also interesting to analyze the properties of particular types of graphs arising in social networks or other type of organizations in which influence spread can take part in the decision process.

Simple games are defined by monotonic families that can be defined succinctly by monotonic functions. Therefore, there are several circuit or graph based formalisms that can be used to represent simple games. Among them the binary decision diagrams (BDDs) has been used as an alternative representation of simple games [11]. This succinct form of representation is most commonly used to represent Boolean functions [1]. BDDs have been used to study several properties of simple games, regular games and weighted games [8]. However, except for some results regarding the computation of power indices, for subfamilies of simple games [11], the complexity of most of the problems studied in this paper remain as an open problem.

Lastly, even though we have shown that all games with polynomial dimension or polynomial codimension can be represented as weighted influence games in polynomial time (i.e., they admit weighted influence graphs with polynomial number of agents), a fundamental open question is determining which simple games can be represented as an (unweighted) influence game with polynomial number of agents. In particular it remains open to know whether 
there are games with exponential dimension that also require an exponential number of players in any representation as influence games. In this line, we know that the simple game with exponential dimension with respect to the players of Section 2 in [28] can be represented by an unweighted influence game in polynomial time with respect to the number of players (see Figure 12). Another candidate is the simple game with exponential dimension of Theorem 8 in [20] for which we have been unable to show whether it can be represented by an (unweighted) influence game with polynomial number of agents.

\section{Acknowledgments}

We thank S. Kurz for pointing out the proof of Theorem 5, and E. Elkind for the proof of Theorem 7. We also thank two anonymous referees for their careful reading and their useful comments and suggestions that helped us to improve the contents and the presentation of the paper.

\section{References}

[1] S. Akers. Binary decision diagrams. IEEE Transactions on Computers, C-27(6):509-516, 1978.

[2] K. R. Apt and E. Markakis. Diffusion in social networks with competing products. In G. Persiano, editor, Algorithmic Game Theory, 4th International Symposium, SAGT 2011, Amalf, Italy, October 17-19, 2011. Proceedings, volume 6982 of Lecture Notes in Computer Science, pages 212-223, 2011.

[3] G. Ausiello, P. Crescenzi, G. Gambosi, V. Kann, A. Marchetti-Spaccamela, and M. Protasi. Complexity and approximation: Combinatorial optimization problems and their approximability properties. Springer-Verlag, Secaucus, NJ, 1999.

[4] H. Aziz. Complexity of comparison of influence of players in simple games. In Ulle Endriss and Paul W. Goldberg, editors, Proceedings of the 2nd International Workshop on Computational Social Choice, (COMSOC 2008), pages 61-72, 2008.

[5] H. Aziz. Algorithmic and complexity aspects of simple coalitional games. PhD thesis, Department of Computer Science, University of Warwick, 2009

[6] Y. Bachrach, D. C. Parkes, and J. S. Rosenschein. Computing cooperative solution concepts in coalitional skill games. Artificial Intelligence, 204:1-21, 2013.

[7] J. F. Banzhaf. Weighted voting doesn't work. Rutgers Law Review, 19:317-343, 1965.

[8] R. Berghammer and S. Bolus. On the use of binary decision diagrams for solving problems on simple games. European Journal of Operation Research, 222(3):529-541, 2012.

[9] S. Bharathi, D. Kempe, and M. Salek. Competitive influence maximization in social networks. In X. Deng and F. C. Graham, editors, Internet and Network Economics, Third International Workshop, WINE 2007, San Diego, CA, USA, December 12-14, 2007, Proceedings, volume 4858 of Lecture Notes in Computer Science, pages 306-311, 2007.

[10] B. Bollobás. Modern graph theory, volume 184 of Graduate Texts in Mathematics. Springer-Verlag, New York, NY, 1998.

[11] S. Bolus. Power indices of simple games and vector-weighted majority games by means of binary decision diagrams. European Journal of Operation Research, 210(2):258-272, 2011.

[12] A. Borodin, Y. Filmus, and J. Oren. Threshold models for competitive influence in social networks. In A. Saberi, editor, Internet and Network Economics - 6th International Workshop, WINE 2010, Stanford, CA, USA, December 13-17, 2010. Proceedings, volume 6484 of Lecture Notes in Computer Science, pages 539-550, 2010.

[13] G. Chalkiadakis, E. Elkind, and M. Wooldridge. Computational aspects of cooperative game theory. Synthesis Lectures on Artificial Intelligence and Machine Learning. Morgan \& Claypool Publishers, 2011.

[14] N. Chen. On the approximability of influence in social networks. SIAM Journal on Discrete Mathematics, 23(3):1400-1415, 2009.

[15] A. Darmann, G. Nicosia, U. Pferschy, and J. Schauer. The subset sum game. European Journal of Operational Research, 233(3):539-549, 2014.

[16] X. Deng and C. Papadimitriou. On the complexity of cooperative solution concepts. Mathematics of Operations Research, 19(2):257-266, 1994.

[17] V. G. Deĭneko and G. J. Woeginger. On the dimension of simple monotonic games. European Journal of Operational Research, 170(1):315318, 2006.

[18] P. Domingos and M. Richardson. Mining the network value of customers. In D. Lee, M. Schkolnick, F. J. Provost, and R. Srikant, editors, Proceedings of the seventh ACM SIGKDD international conference on Knowledge discovery and data mining, San Francisco, CA, USA, August 26-29, 2001, pages 57-66, 2001.

[19] D. Easley and J. Kleinberg. Networks, crowds and markets: Reasoning about a highly connected world. Cambridge University Press, New York, NY, 2010.

[20] E. Elkind, L. A. Goldberg, P. W. Goldberg, and M. Wooldridge. On the dimensionality of voting games. In D. Fox and C. P. Gomes, editors, Proceedings of the Twenty-Third AAAI Conference on Artificial Intelligence, AAAI 2008, Chicago, Illinois, USA, July 13-17, 2008, pages 69-74, 2008

[21] E. Even-Dar and A. Shapira. A note on maximizing the spread of influence in social networks. Information Processing Letters, 111(4):184$187,2011$.

[22] P. Faliszewski, E. Elkind, and M. Wooldridge. Boolean combinations of weighted voting games. In C. Sierra, C. Castelfranchi, K. S. Decker, and J. S. Sichman, editors, 8th International Joint Conference on Autonomous Agents and Multiagent Systems (AAMAS 2009), Budapest, Hungary, May 10-15, 2009, Volume 1, pages 185-192, 2009. 
[23] M. Fredman and L. Khachiyan. On the complexity of dualization of monotone disjunctive normal forms. Journal of Algorithms, 21(3):618628, 1996.

[24] J. Freixas. Power indices. In J. J. Cochran, L. A. Cox, P. Keskinocak, J. P. Kharoufeh, and J. C. Smith, editors, Wiley Encyclopedia of Operations Research and Management Science, volume 8. John Wiley \& Sons, 2011.

[25] J. Freixas and D. Marciniak. A minimum dimensional class of simple games. TOP: An Official Journal of the Spanish Society of Statistics and Operations Research, 17(2):407-414, 2009.

[26] J. Freixas and X. Molinero. On the existence of a minimum integer representation for weighted voting systems. Annals of Operation Research, 166(1):243-260, 2009.

[27] J. Freixas, X. Molinero, M. Olsen, and M. Serna. On the complexity of problems on simple games. RAIRO-Operations Research, 45(4):295$314,2011$.

[28] J. Freixas and M. A. Puente. A note about games-composition dimension. Discrete Applied Mathematics, 113(2-3):265-273, 2001.

[29] M. R. Garey and D. S. Johnson. Computers and intractability: A guide to the theory of NP-Completness. A Series of Books in the Mathematical Sciences. W. H. Freeman and Company, New York, NY, 1979.

[30] D. B. Gillies. Some theorems on n-person games. PhD thesis, Department of Mathematics, Princeton University, 1953.

[31] J. Goldenberg, B. Libai, and E. Muller. Talk of the network: A complex systems look at the underlying process of word-of-mouth. Marketing Letters, 12(3):211-223, 2001.

[32] J. Goldenberg, B. Libai, and E. Muller. Using complex systems analysis to advance marketing theory development: Modeling heterogeneity effects on new product growth through stochastic cellular automata. Academy of Marketing Science Review, 5(9), 2001.

[33] M. Granovetter. Threshold models of collective behavior. American Journal of Sociology, 83(6):1420-1443, 1978.

[34] T. Hellmann and M. Staudigl. Evolution of social networks. European Journal of Operational Research, 234(3):583-596, 2014.

[35] S. Hu. Threshold logic. University of California Press, Berkeley and Los Angeles, CA, 1965.

[36] M. T. Irfan and L. E. Ortiz. A game-theoretic approach to influence in networks. In W. Burgard and D. Roth, editors, Proceedings of the Twenty-Fifth AAAI Conference on Artificial Intelligence, AAAI 2011, San Francisco, California, USA, August 7-11, 2011, pages 688-694, 2011.

[37] M. Jackson. Social and economic networks. Princeton University Press, Princeton, NJ, 2008.

[38] R. G. Jeroslow. On defining sets of vertices of the hypercube by linear inequalities. Discrete Mathematics, 11(2):119-124, 1975.

[39] D. Kempe, J. Kleinberg, and É. Tardos. Maximizing the spread of influence through a social network. In L. Getoor, T. E. Senator, P. Domingos, and C. Faloutsos, editors, Proceedings of the Ninth ACM SIGKDD International Conference on Knowledge Discovery and Data Mining, Washington, DC, USA, August 24 - 27, 2003, pages 137-146, 2003.

[40] D. Kleitman and G. Markowsky. On Dedekind's problem: the number of isotone boolean functions. II. Transactions of the American Mathematical Society, 213:373-390, 1975.

[41] E. M. Luks. Hypergraph isomorphism and structural equivalence of boolean functions. In J. S. Vitter, L. L. Larmore, and F. T. Leighton, editors, Proceedings of the Thirty-First Annual ACM Symposium on Theory of Computing, May 1-4, 1999, Atlanta, Georgia, USA, pages 652-658, 1999.

[42] T. Matsui and Y. Matsui. A survey of algorithms for calculating power indices of weighted majority games. Journal of the Operations Research Society of Japan, 43(1):71-86, 2000.

[43] L. Monroy and F. R. Fernández. The Shapley-Shubik index for multi-criteria simple games. European Journal of Operational Research, 209(2):122-128, 2011.

[44] C. Papadimitriou. Computational complexity. Addison-Wesley, Reading, MA, 1994.

[45] I. Parberry. Circuit complexity and neural networks. Foundations of Computers. MIT Press, Cambridge, MA, 1994.

[46] A. Polyméris. Stability of two player game structures. Discrete applied mathematics, 156(14):2636-2646, 2008.

[47] A. Polyméris, R. Contreras, M. A. Pinninghoff, and E. Osses. Response-ability and its complexity. In H. R. Arabnia and Y. Mun, editors, Proceedings of the International Conference on Artificial Intelligence, IC-AI '02, June 24 - 27, 2002, Las Vegas, Nevada, USA, Volume 3, pages 1239-1245, 2002.

[48] K. G. Ramamurthy. Coherent structures and simple games, volume 6 of Theory and Decision Library: Game Theory, Mathematical Programming and Operations Research. Kluwer Academic Publishers, Dordrecht, Netherlands, 1990.

[49] M. Richardson and P. Domingos. Mining knowledge-sharing sites for viral marketing. In Proceedings of the Eighth ACM SIGKDD International Conference on Knowledge Discovery and Data Mining, July 23-26, 2002, Edmonton, Alberta, Canada, pages 61-70, 2002.

[50] F. Riquelme and A. Polyméris. On the complexity of the decisive problem in simple and weighted games. Electronic Notes in Discrete Mathematics, 37:21-26, 2011.

[51] T. Schelling. Micromotives and macrobehavior. Fels lectures on public policy analysis. W. W. Norton \& Company, New York, NY, 1978.

[52] A. Taylor and W. Zwicker. Weighted voting, multicameral representation, and power. Games and Economic Behavior, 5(1):170-181, 1993.

[53] A. Taylor and W. Zwicker. Simple games: Desirability relations, trading, pseudoweightings. Princeton University Press, Princeton, NJ, 1999.

[54] J. von Neumann and O. Morgenstern. Theory of games and economic behavior. Princeton University Press, Princeton, NJ, 1944.

[55] M. Wooldridge and P. E. Dunne. On the computational complexity of qualitative coalitional games. Artificial Intelligence, 158(1):27-73, 2004.

[56] M. Wooldridge and P. E. Dunne. On the computational complexity of coalitional resource games. Artificial Intelligence, 170(10):835-871, 2006. 